\title{
Comparing EFT and exact one-loop analyses of non-degenerate stops
}

\author{
Aleksandra Drozd, ${ }^{a}$ John Ellis, ${ }^{a, b}$ Jérémie Quevillon ${ }^{a}$ and Tevong You ${ }^{a}$ \\ ${ }^{a}$ Theoretical Particle Physics and Cosmology Group, Physics Department, King's College London, \\ London WC2R 2LS, U.K. \\ ${ }^{b}$ TH Division, Physics Department, CERN, \\ CH-1211 Geneva 23, Switzerland \\ E-mail: aleksandra.drozd@kcl.ac.uk, john.ellis@kcl.ac.uk, \\ jeremie.quevillon@kcl.ac.uk, tevong.you@kcl.ac.uk
}

ABSTRACT: We develop a universal approach to the one-loop effective field theory (EFT) using the Covariant Derivative Expansion (CDE) method. We generalise previous results to include broader classes of UV models, showing how expressions previously obtained assuming degenerate heavy-particle masses can be extended to non-degenerate cases. We apply our method to the general MSSM with non-degenerate stop squarks, illustrating our approach with calculations of the coefficients of dimension- 6 operators contributing to the $h g g$ and $h \gamma \gamma$ couplings, and comparing with exact calculations of one-loop Feynman diagrams. We then use present and projected future sensitivities to these operator coefficients to obtain present and possible future indirect constraints on stop masses. The current sensitivity is already comparable to that of direct LHC searches, and future FCC-ee measurements could be sensitive to stop masses above a TeV. The universality of our one-loop EFT approach facilitates extending these constraints to a broader class of UV models.

Keywords: Supersymmetry Phenomenology, Phenomenology of Field Theories in Higher Dimensions

ARXIV EPRINT: 1504.02409 


\section{Contents}

1 Introduction 1

2 The covariant derivative expansion and the one-loop effective action 3

2.1 The non-degenerate one-loop effective Lagrangian 3

$\begin{array}{ll}2.2 & \text { A light stop in the } h g g \text { and } h \gamma \gamma \text { couplings }\end{array}$

3 Feynman diagram calculations and comparison $\quad 8$

4 Constraints on light stops from a global fit $\quad 11$

$\begin{array}{lll}4.1 & \text { Degenerate stop masses } & 13\end{array}$

$\begin{array}{lll}4.2 & \text { Non-degenerate stop masses } & 15\end{array}$

5 Sensitivities of possible future precision measurements $\quad 17$

$\begin{array}{lll}5.1 & \text { Degenerate stop masses } & 18\end{array}$

$\begin{array}{lll}5.2 & \text { Non-degenerate stop masses } & 19\end{array}$

6 Conclusions and prospects $\quad 20$

\section{Introduction}

In view of the overall consistency between the current measurements of particle properties and predictions in the Standard Model (SM), a common approach to the analysis of present and prospective future data is to describe them via an effective field theory (EFT) in which the renormalizable SM $d=4$ Lagrangian is supplemented with higher-dimensional terms composed from SM fields [1-7]. To the extent that this new physics has a mass scale that is substantially higher than the energy scale of the available measurements [8], the EFT approach is a powerful way to constrain possible new physics beyond the SM (BSM) that is model-independent [9-18]. The $d=6$ operators in this Effective SM (ESM) were first classified in $[1],{ }^{1}$ with a complete basis using equations of motion to eliminate redundancies [2-7] being first presented in [21]. There have been many studies of various aspects of these dimension- 6 operators, ${ }^{2}$ and a short review can be found in [76].

The EFT approach may well be a good approximation if the new physics affects precision observables at the tree level, or if it is strongly-interacting. In these cases the new physics mass scale is likely to be relatively high, and considering the lowest-dimensional

\footnotetext{
${ }^{1}$ This EFT approach that we follow, in which the $\mathrm{SU}(2)_{L} \times \mathrm{U}(1)_{Y}$ electroweak symmetry is linearly realized, is to be distinguished from a non-linear EFT based on the chiral electroweak Lagrangian [19] and the more general anomalous coupling framework of a U(1) EM effective Lagrangian [20].

${ }^{2}$ See $[1-7,22-27]$ for some examples of earlier work and [9-18, 21, 28-75] for a sampling of more recent studies.
} 
EFT operators may well be sufficient. However, the EFT approach may have limitations if the new physics has effects only at the loop level, or is weakly interacting. In these cases, the EFT approach may be sensitive only to new physics at some relatively low mass scale, and the new physics effects may not be characterised well by considering simply the lowest-dimensional EFT operators.

Examples in the first, 'safer' category may include certain models with extended Higgs sectors [75], such as two-Higgs-doublet models, or some composite models. Examples in the second category may include the loop effects of supersymmetric models. However, even in this case it is possible that precision electroweak and Higgs data may provide interesting constraints on the possible masses of stop squarks, which have relatively large Yukawa couplings to the SM Higgs field. In particular, the EFT approach may be useful in the framework of 'natural' supersymmetric models with stops that have masses above $100 \mathrm{GeV}$ but still relatively light compared to other supersymmetric particles.

Important steps towards the calculation of loop effects and the simplification of their matching with EFT coefficients have been taken recently by Henning, Lu and Murayama (HLM) $[72,73]$. In particular, they use a covariant-derivative expansion (CDE) $[77,78]$ to characterise new-physics effects via the evaluation of the one-loop effective action. They apply these techniques to derive universal results and also study some explicit models including electroweak triplet scalars, an extra electroweak scalar doublet, and light stops within the minimal supersymmetric extension of the SM (MSSM), as well as some other models. They also discuss electroweak precision observables, triple-gauge couplings and Higgs decay widths and production cross sections [73], and have used their results to derive indicative constraints on the basis of present and future data [72].

In this paper we discuss aspects of the applicability of the EFT approach to models with relatively light stops, exploring in more depth some issues arising from the work of HLM $[72,73]$. As they discuss, using the CDE and the one-loop effective action is more elegant and less time-consuming than a complete one-loop Feynman diagram computation. On the other hand, they applied their approach to models with degenerate soft supersymmetry-breaking terms for the stop squarks, and we show how to extend their approach to the non-degenerate case, with specific applications to the dimension-6 operators that contribute to the $h g g$ and $h \gamma \gamma$ couplings. Our extension of the CDE approach would also permit applications to a wider class of ultra-violet (UV) extensions of the SM and other EFT operators.

Another important aspect of our work is a comparison of the EFT results with the corresponding full one-loop Feynman diagram calculations also in the non-degenerate case, so as to assess the accuracy of the EFT approach for analysing present and future data.

In a recent paper, together with Sanz, two of us (JE and TY) made a global fit to dimension-6 EFT operator coefficients including electroweak precision data, LHC measurements of triple-gauge couplings, Higgs rates and production kinematics [18]. Here we use this global fit to constrain the stop mass $m_{\tilde{t}}$ and the mixing parameter $X_{t}$, comparing results obtained using the EFT with those using the full one-loop diagrammatic calculation. The bounds on $m_{\tilde{t}}$ and $X_{t}$ are strongly correlated, and we find that the EFT approach may yield quite accurate constraints for the limits of larger $m_{\tilde{t}}$ and $X_{t}$. However, there 
are substantial differences from the full diagrammatic result for smaller $m_{\tilde{t}}$ and $X_{t}$. In this case the diagrammatic approach gives indirect constraints on the stop squark that are quite competitive with direct experimental searches at the LHC. We also explore the possible accuracy of the EFT for possible future data sets, including those obtainable from the LHC and possible $e^{+} e^{-}$colliders. ${ }^{3}$ For example, possible FCC-ee measurements [83] may be sensitive indirectly to stop masses $\gtrsim 1 \mathrm{TeV}$.

The layout of this paper is as follows. In section 2 we introduce the covariant derivative expansion (CDE) and discuss its application to the one-loop effective action, highlighting how the HLM approach [72, 73] may be extended to the case of non-degenerate squarks. As we discuss, one way to achieve this is to use the Baker-Campbell-Hausdorff (BCH) theorem to rearrange the one-loop effective action, and another is to introduce an auxiliary expansion variable. Results obtained by these two methods agree, and are also consistent with the full one-loop Feynman diagram result presented in section 3. Analyses of the current data in the frameworks of the EFT and the diagrammatic approach are presented in section 4, and their results compared. Studies of the possible sensitivities of future measurements at the ILC and FCC-ee are presented in section 5, and section 6 discusses our conclusions and possible directions for future work.

\section{The covariant derivative expansion and the one-loop effective action}

The one-loop effective action may be obtained by integrating out directly the heavy particles in the path integral using the saddle-point approximation of the functional integral. The contributions to operators involving only light fields can be evaluated by various expansion methods for the application of the path integral. Here we follow the Covariant Derivative Expansion (CDE), a manifestly gauge-invariant method first introduced in the 1980s by Gaillard [77] and Cheyette [78], and recently applied to the Effective SM (ESM) by Henning, Lu and Murayama (HLM) [73]. ${ }^{4}$ The latter provide, in particular, universal results for operators up to dimension- 6 in the form of a one-loop effective Lagrangian with coefficients evaluated via momentum integrals. This approach applies generally, and greatly simplifies the matching to UV models, since it avoids the necessity of recalculating one-loop Feynman diagrams for every model. However, HLM assume a degenerate mass matrix, which may not be the case in general, as for example in the 'natural' MSSM with light stops. We show here how their results may be extended to the non-degenerate case for the one-loop effective Lagrangian terms involved in the dimension- 6 operators affecting the $h g g$ and $h \gamma \gamma$ couplings, with application to the case of non-degenerate stops and sbottoms.

\subsection{The non-degenerate one-loop effective Lagrangian}

We consider a generic Lagrangian consisting of the SM part with complex heavy scalar fields arranged in a multiplet $\Phi$,

$$
\mathcal{L}_{\mathrm{UV}}=\mathcal{L}_{\mathrm{SM}}+\left(\Phi^{\dagger} F(x)+\text { h.c. }\right)+\Phi^{\dagger}\left(P^{2}-M^{2}-U(x)\right) \Phi+\mathcal{O}\left(\Phi^{3}\right),
$$

\footnotetext{
${ }^{3}$ For previous analyses, see [72, 79-81].

${ }^{4}$ We thank Hermès Bélusca-Maïto for pointing out to us another recent paper that computes the one-loop effective action for certain dimension-6 QCD operators [82].
} 
where $P \equiv i D_{\mu}$, with $D_{\mu}$ the gauge-covariant derivative, $F(x)$ and $U(x)$ are combinations of SM fields coupling linearly and quadratically respectively to $\Phi$, and $M$ is a diagonal mass matrix. The path integral over $\Phi$ may be computed by expanding the action around the minimum with respect to $\Phi$, so that the linear terms give the tree-level effective Lagrangian upon substituting the equation of motion for $\Phi$ :

$$
\mathcal{L}_{\text {tree }}^{\text {eff }}=\sum_{n=0} F^{\dagger} M^{-2}\left[\left(P^{2}-U\right) M^{-2}\right]^{n} F+\mathcal{O}\left(\Phi^{3}\right)
$$

whereas the quadratic terms are responsible for the one-loop part of the effective Lagrangian. After evaluating the functional integral and Fourier transforming to momentum space, this can be written in the form

$$
\mathcal{L}_{1-\text { loop }}^{\text {eff }}=i \int \frac{d^{4} q}{(2 \pi)^{4}} \operatorname{Tr} \ln \left(-\left(P_{\mu}-q_{\mu}\right)^{2}+M^{2}+U\right) .
$$

It is convenient, before expanding the logarithm, to shift the momentum using the covariant derivative, by inserting factors of $e^{ \pm P_{\mu} \partial / \partial q_{\mu}}$ :

$$
\mathcal{L}_{1 \text {-loop }}^{\text {eff }}=i \int \frac{d^{4} q}{(2 \pi)^{4}} \operatorname{Tr} \ln \left[e^{P_{\mu} \partial / \partial q_{\mu}}\left(-\left(P_{\mu}-q_{\mu}\right)^{2}+M^{2}+U\right) e^{-P_{\mu} \partial / \partial q_{\mu}}\right] .
$$

This choice ensures a convergent expansion while the calculation of operators remains manifestly gauge-invariant throughout. ${ }^{5}$ The result is a series involving gauge field strengths, covariant derivatives and SM fields encoded in the matrix $U(x)$ :

$$
\mathcal{L}_{1-\text { loop }}^{\text {eff }}=i \int \frac{d^{4} q}{(2 \pi)^{4}} \operatorname{Tr} \ln \left[-\left(\tilde{G}_{\nu \mu} \partial / \partial q_{\mu}+q_{\mu}\right)^{2}+M^{2}+\tilde{U}\right]
$$

where

$$
\begin{aligned}
\tilde{G}_{\nu \mu} & \equiv \sum_{n=0} \frac{n+1}{(n+2) !}\left[P_{\alpha_{1}},\left[\ldots\left[P_{\alpha_{n}}, G_{\nu \mu}^{\prime}\right]\right]\right] \frac{\partial^{n}}{\partial q_{\alpha_{1}} \ldots q_{\alpha_{n}}}, \\
\tilde{U} & =\sum_{n=0} \frac{1}{n !}\left[P_{\alpha_{1}},\left[\ldots\left[P_{\alpha_{n}}, U\right]\right]\right] \frac{\partial^{n}}{\partial q_{\alpha_{1}} \ldots q_{\alpha_{n}}}
\end{aligned}
$$

Here we defined $G_{\nu \mu}^{\prime} \equiv-i G_{\nu \mu}$ with the field strength given by $\left[P_{\nu}, P_{\mu}\right]=-G_{\nu \mu}^{\prime}$. It is convenient to group together the terms involving momentum derivatives:

$$
\mathcal{L}_{1-\text { loop }}^{\text {eff }}=i \int \frac{d^{4} q}{(2 \pi)^{4}} \operatorname{Tr} \ln (A+B),
$$

where

$$
\begin{aligned}
A & \equiv-\left\{q_{\mu}, \tilde{G}_{\nu \mu}\right\} \frac{\partial}{\partial q_{\nu}}-\tilde{G}_{\nu \mu} \tilde{G}_{\alpha \mu} \frac{\partial^{2}}{\partial q_{\nu} q_{\alpha}}+\delta \tilde{U} \\
B & \equiv-q^{2}+M^{2}+U
\end{aligned}
$$

and we have separated $\tilde{U}=U+\delta \tilde{U}$.

\footnotetext{
${ }^{5}$ We refer the reader to $[73,77,78]$ for technical details and discussions of the CDE method.
} 
Expanding the logarithm using the Baker-Campbell-Hausdorff (BCH) formula gives $\ln (A+B)=\ln (B)+\ln \left(1+B^{-1} A\right)+\frac{1}{2}\left[\ln B, \ln \left(1+B^{-1} A\right)\right]+\frac{1}{12}\left[\ln B,\left[\ln B, \ln \left(1+B^{-1} A\right)\right]\right]+\ldots$ and, using the identity $[\ln X, Y]=\sum_{n=1} \frac{1}{n} X^{-n} L_{X}^{n} Y$, where $L_{X} Y \equiv[X, Y]$, we see that all possible gauge-invariant operators are obtained by evaluating commutators of $A$ and $B$.

As an example, we compute the term contributing to the dimension- 6 operator affecting Higgs production by gluon fusion:

$$
\mathcal{O}_{g}=g_{3}^{2}\left|H^{2}\right| G_{\mu \nu}^{a} G^{a \mu \nu} .
$$

The calculation can be organised by writing $A$ as a series in momentum derivatives,

$$
A=\sum_{n=1} A_{n}^{\alpha_{1} \ldots \alpha_{n}} \frac{\partial^{n}}{\partial q_{\alpha_{1}} \ldots \partial q_{\alpha_{n}}}=A_{1}^{\alpha_{1}} \frac{\partial}{\partial q_{\alpha_{1}}}+A_{2}^{\alpha_{1} \alpha_{2}} \frac{\partial^{2}}{\partial q_{\alpha_{1} q_{\alpha_{2}}}}+\ldots,
$$

where each term is obtained by substituting $\tilde{G}$ and $\tilde{U}$ in eq. (2.2). Here we require only the part $A_{2}^{\alpha_{1} \alpha_{2}} \supset-\frac{1}{4} G_{\alpha_{1} \mu}^{\prime} G_{\alpha_{2} \mu}^{\prime}$, together with the following commutators:

$$
i \int \frac{d^{4} q}{(2 \pi)^{4}} \operatorname{Tr} \ln (A+B) \supset i \int \frac{d^{4} q}{(2 \pi)^{4}} \operatorname{Tr}\left(\frac{1}{2} B^{-2}[B, A]+\frac{1}{3} B^{-3}[B,[B, A]]\right) .
$$

We note that $M$ and $U$ are $n \times n$ matrices that do not commute in general, which motivates the use of the $\mathrm{BCH}$ expansion, first applied to the CDE in [78]. Evaluating the commutators we find

$$
\mathcal{L}_{1 \text {-loop }}^{\text {eff }} \supset i \int \frac{d^{4} q}{(2 \pi)^{4}} \operatorname{Tr}\left\{B^{-2}\left(-\frac{1}{4} G_{\nu \mu}^{\prime} G^{\prime \nu \mu}\right)+\frac{8}{3} q_{\alpha} q_{\nu} B^{-3}\left(-\frac{1}{4} G_{\mu}^{\prime \alpha} G^{\prime \nu \mu}\right)\right\}
$$

and using $B^{-1}=-\Delta \sum_{n=0}(\Delta U)^{n}$, where $\Delta \equiv 1 /\left(q^{2}-M^{2}\right)$, we see that to obtain operators up to dimension 6 requires retaining up to two powers of $U$, so that we have traces of the form

$$
\begin{aligned}
& \operatorname{Tr}\left(\Delta^{a} U G^{\prime \alpha}{ }_{\mu} G^{\prime \nu \mu}\right)=\sum_{i=1}^{n}\left(\Delta_{i}^{a} U_{i i} G_{i}^{\prime \alpha}{ }_{\mu} G_{i}^{\nu \mu}\right), \\
& \operatorname{Tr}\left(\Delta^{a} U \Delta^{b} U \Delta^{c} G^{\prime \alpha}{ }_{\mu} G^{\prime \nu \mu}\right)=\sum_{i=1}^{n} \sum_{j=1}^{n}\left(\Delta_{i}^{a+c} \Delta_{j}^{b} U_{i j} U_{j i} G_{i}^{\prime \alpha}{ }_{\mu}^{\prime \nu \mu}\right) .
\end{aligned}
$$

Here we assume $G^{\prime}=\operatorname{diag}\left(G_{1}^{\prime}, \ldots, G_{n}^{\prime}\right)$ and $\Delta=\operatorname{diag}\left(\Delta_{1}, \ldots, \Delta_{n}\right)$, where $\Delta_{i} \equiv 1 /\left(q^{2}-m_{i}^{2}\right)$, and $U$ is a general $n \times n$ matrix. To evaluate the momentum integrals of arbitrary powers of mixed propagators we need to combine them using Feynman parameters:

$$
\int \frac{d^{4} q}{(2 \pi)^{4}} q^{l} \Delta_{i}^{a} \Delta_{j}^{b}=\frac{(a+b+1) !}{(a-1) !(b-1) !} \int_{0}^{1} d z_{i} d z_{j}\left[z_{i}^{a-1} z_{j}^{b-1}\left(\int \frac{d^{4} q}{(2 \pi)^{4}} q^{l} \Delta_{i j}^{a+b}\right) \delta\left(1-z_{i}-z_{j}\right)\right],
$$

where $\Delta_{i j} \equiv 1 /\left(q^{2}-m_{i}^{2} z_{i}-m_{j}^{2} z_{j}\right)$. Taking care in applying the $\delta$-function in the summation over the matrix indices, we finally obtain the following expression valid in the case of a non-degenerate mass matrix:

$$
\mathcal{L}_{1 \text {-loop }}^{\text {eff }} \supset \frac{1}{(4 \pi)^{2}}\left[-\frac{1}{12} \sum_{i=1}^{n}\left(\frac{U_{i i}}{m_{i}^{2}} G_{i \mu \nu}^{\prime} G_{i}^{\prime \mu \nu}\right)+\frac{1}{24} \sum_{i=1}^{n} \sum_{j=1}^{n}\left(\frac{U_{i j} U_{j i}}{m_{i}^{2} m_{j}^{2}} G_{i \mu \nu}^{\prime} G_{i}^{\prime \mu \nu}\right)\right] .
$$


We have checked this result by extending the log-expansion method of [73] to the nondegenerate case by introducing an auxiliary parameter $\xi$ and then differentiating under the integral sign:

$$
\begin{aligned}
\mathcal{L}_{1 \text { loop }}^{\text {eff }} & =i \int \frac{d^{4} q}{(2 \pi)^{4}} \operatorname{Tr} \ln \left[-\left(\tilde{G}_{\nu \mu} \partial / \partial q_{\mu}+q_{\mu}\right)^{2}+\xi M^{2}+\tilde{U}\right] \\
& =i \int \frac{d^{4} q}{(2 \pi)^{4}} \int d \xi \operatorname{Tr}\left(\frac{1}{A+U-\Delta_{\xi}^{-1}} M^{2}\right)
\end{aligned}
$$

where $\Delta^{\xi} \equiv 1 /\left(q^{2}-\xi M^{2}\right)$ and $\xi$ is set to 1 at the end of the calculation. The expansion then reads

$$
\mathcal{L}_{1 \text { loop }}^{\text {eff }}=i \int \frac{d^{4} q}{(2 \pi)^{4}} \int d \xi \operatorname{Tr}\left\{\sum_{n=0}^{\infty}\left[-\Delta^{\xi}(A+U)\right]^{n} \Delta^{\xi} M^{2}\right\}
$$

and yields the same result as in (2.3), demonstrating the consistency of our approach.

In general the field strength matrix $G_{\mu \nu}$ may not be diagonal, as for example when the $\Phi$ multiplet contains an $\mathrm{SU}(2)_{L}$ doublet and singlet, so that we have a $2 \times 2$ non-diagonal sub-matrix $W_{\mu \nu}^{a} \tau^{a}$ involving the weak gauge bosons $W_{\mu}^{a}$. The relevant non-degenerate one-loop effective Lagrangian terms then generalise to the universal expression

$$
\mathcal{L}_{1 \text {-loop }}^{\text {eff }} \supset \frac{1}{(4 \pi)^{2}}\left\{-\frac{1}{12} \operatorname{Tr}\left(\bar{U} G_{\mu \nu}^{\prime} G^{\prime \mu \nu}\right)+\frac{1}{24} \operatorname{Tr}\left(\bar{U}^{2} G_{\mu \nu}^{\prime} G^{\prime \mu \nu}\right)+\frac{1}{240} \operatorname{Tr}\left(\left[\bar{U}, G_{\mu \nu}^{\prime}\right]\left[\bar{U}, G^{\prime \mu \nu}\right]\right)\right\},
$$

where $\bar{U}_{i j} \equiv \frac{U_{i j}}{m_{i} m_{j}}$, which is sufficient for computing the one-loop coefficients in the $h g g$ and $h \gamma \gamma$ couplings. ${ }^{6}$

\subsection{A light stop in the $h g g$ and $h \gamma \gamma$ couplings}

The result (2.4) is universal in the sense that all the UV information is encapsulated in the $U, M$ matrices and the $P_{\mu}$ covariant derivative, while the operator coefficients are determined by integrals over momenta that are performed once and for all. The simplicity of this approach is illustrated by integrating out stops in the MSSM, whose leading-order contribution necessarily appears at one-loop due to R-parity. Since gluon fusion in the SM also occurs at one-loop and currently provides the strongest constraint on any dimension6 operator in the Higgs sector, we first calculate its Wilson coefficient within the EFT framework. Later we extend the calculation to the the dimension- 6 operators contributing to the $h \gamma \gamma$ coupling, and comment on the extension to other dimension- 6 operators.

The $M$ and $U$ matrices are given by the quadratic stop term in the MSSM Lagrangian,

$$
\mathcal{L}_{\mathrm{MSSM}} \supset \Phi^{\dagger}\left(M^{2}+U(x)\right) \Phi
$$

\footnotetext{
${ }^{6}$ We provide more details on obtaining this and the rest of the non-degenerate universal one-loop effective Lagrangian in a forthcoming work in preparation [84].
} 
where $\Phi=\left(\tilde{Q}, \tilde{t}_{R}^{*}\right)$, and

$$
\begin{aligned}
M^{2} & =\left(\begin{array}{cc}
m_{\tilde{Q}}^{2} & 0 \\
0 & m_{\tilde{t}_{R}}^{2}
\end{array}\right) \\
U & =\left(\begin{array}{cc}
\left(h_{t}^{2}+\frac{1}{2} g_{2}^{2} c_{\beta}^{2}\right) \tilde{H} \tilde{H}^{\dagger}+\frac{1}{2} g_{2}^{2} s_{\beta}^{2} H H^{\dagger}-\frac{1}{2}\left(g_{1}^{2} Y_{\tilde{Q}} c_{2 \beta}+\frac{1}{2} g_{2}^{2}\right)|H|^{2} & h_{t} X_{t} \tilde{H} \\
h_{t} X_{t} \tilde{H}^{\dagger} & \left(h_{t}^{2}-\frac{1}{2} g_{1}^{2} Y_{\tilde{t}_{R}} c_{2 \beta}\right)|H|^{2}
\end{array}\right) .
\end{aligned}
$$

Here we have defined $\tilde{H} \equiv i \sigma^{2} H^{*}, h_{t} \equiv y_{t} s_{\beta}, X_{t} \equiv A_{t}-\mu \cot \beta$, and the hypercharges are $Y_{\tilde{Q}}=1 / 6, Y_{\tilde{t}_{R}}=-2 / 3$. The mass matrix entries $m_{\tilde{Q}}$ and $m_{\tilde{t}_{R}}$ are the soft supersymmetrybreaking masses in the MSSM Lagrangian. We note that $\tilde{Q}=\left(\tilde{t}_{L}, \tilde{b}_{L}\right)$ is an $\operatorname{SU}(2)_{L}$ doublet, so $U$ is implicitly a $3 \times 3$ matrix, and there will be an additional trace over color. Substituting this into (2.4) with $G_{\mu \nu}$ the gluon field strength, we extract from the universal one-loop effective action the term

$$
\mathcal{L}_{1 \text {-loop }}^{\text {eff }} \supset \frac{1}{(4 \pi)^{2}} \frac{1}{24}\left(\frac{h_{t}^{2}-\frac{1}{6} g_{1}^{2} c_{2 \beta}}{m_{\tilde{Q}}^{2}}+\frac{h_{t}^{2}+\frac{1}{3} g_{1}^{2} c_{2 \beta}}{m_{\tilde{t}_{R}}^{2}}-\frac{h_{t}^{2} X_{t}^{2}}{m_{\tilde{Q}}^{2} m_{\tilde{t}_{R}}^{2}}\right) g_{3}^{2}|H|^{2} G_{\mu \nu}^{a} G^{a \mu \nu} .
$$

This yields the dimension-6 operator $\mathcal{O}_{g}$ in the ESM:

$$
\mathcal{L}_{\operatorname{dim}-6} \supset \frac{\bar{c}_{g}}{m_{W}^{2}} \mathcal{O}_{g}
$$

with the Wilson coefficient given in this normalisation ${ }^{7}$ by

$$
\bar{c}_{g}=\frac{m_{W}^{2}}{(4 \pi)^{2}} \frac{1}{24}\left(\frac{h_{t}^{2}-\frac{1}{6} g_{1}^{2} c_{2 \beta}}{m_{\tilde{Q}}^{2}}+\frac{h_{t}^{2}+\frac{1}{3} g_{1}^{2} c_{2 \beta}}{m_{\tilde{t}_{R}}^{2}}-\frac{h_{t}^{2} X_{t}^{2}}{m_{\tilde{Q}}^{2} m_{\tilde{t}_{R}}^{2}}\right) .
$$

This example demonstrates the relative ease with which one may obtain a Wilson coefficient at the one-loop level without having to compute Feynman diagrams in both the UV model and the EFT that then have to be matched, a process that must be redone every time one adds a new particle to integrate out. Here we may add a right-handed sbottom simply by enlarging the $U$ matrix for $\Phi=\left(\tilde{Q}, \tilde{t}_{R}^{*}, \tilde{b}_{R}^{*}\right)$ and plugging it back into (2.4), giving the result

$\bar{c}_{g}=\frac{m_{W}^{2}}{(4 \pi)^{2}} \frac{1}{24}\left(\frac{h_{b}^{2}+h_{t}^{2}-\frac{1}{6} g_{1}^{2} c_{2 \beta}}{m_{\tilde{Q}^{2}}}+\frac{h_{t}^{2}+\frac{1}{3} g_{1}^{2} c_{2 \beta}}{m_{\tilde{t}_{R}^{2}}}+\frac{h_{b}^{2}-\frac{1}{6} g_{1}^{2} c_{2 \beta}}{m_{\tilde{b}_{R}^{2}}^{2}}-\frac{h_{t}^{2} \tilde{X}_{t}^{2}}{m_{\tilde{Q}^{2}} m_{\tilde{t}_{R}^{2}}}-\frac{h_{b}^{2} \tilde{X}_{b}^{2}}{m_{\tilde{Q}^{2}} m_{\tilde{b}_{R}^{2}}}\right)$.

We compute similarly the dimension- 6 operators affecting the $h \gamma \gamma$ coupling, with the field strength matrix given in this case by

$$
G_{\mu \nu}^{\prime}=\left(\begin{array}{cc}
W_{\mu \nu}^{\prime a} \tau^{a}+Y_{\tilde{Q}} B_{\mu \nu}^{\prime} \mathbb{1} & 0 \\
0 & -Y_{\tilde{t}_{R}} B_{\mu \nu}^{\prime}
\end{array}\right)
$$

\footnotetext{
${ }^{7}$ In general, barred coefficients are related to unbarred ones by $\bar{c} \equiv c \frac{M^{2}}{\Lambda^{2}}$ where $M=v, m_{W}$ depending on the operator normalisation in the Lagrangian.
} 

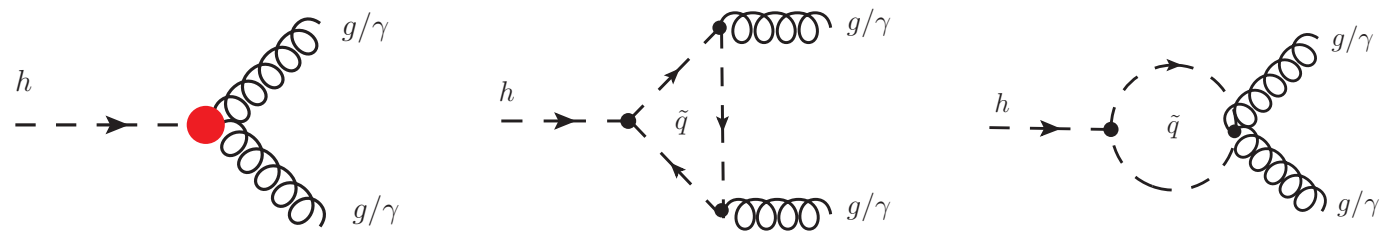

Figure 1. Leading order tree-level Feynman diagram for the EFT (left) and one-loop diagrams for the squark contributions (middle and right) to the $h \rightarrow g g / \gamma \gamma$ amplitude.

Evaluating (2.4) then yields directly

$$
\mathcal{L}_{\operatorname{dim}-6} \supset \frac{\bar{c}_{B B}}{m_{W}^{2}} \mathcal{O}_{B B}+\frac{\bar{c}_{W W}}{m_{W}^{2}} \mathcal{O}_{W W}+\frac{\bar{c}_{W B}}{m_{W}^{2}} \mathcal{O}_{W B},
$$

where

$$
\mathcal{O}_{B B}=g_{1}^{2}|H|^{2} B_{\mu \nu} B^{\mu \nu}, \quad \mathcal{O}_{W W}=g_{2}^{2}|H|^{2} W_{\mu \nu}^{a} W^{a \mu \nu}, \quad \mathcal{O}_{W B}=2 g_{1} g_{2} H^{\dagger} \tau^{a} H W_{\mu \nu}^{a} B^{\mu \nu},
$$

and

$$
\begin{aligned}
\bar{c}_{B B}= & \frac{m_{W}^{2}}{(4 \pi)^{2}}\left(\frac{1}{144} \frac{\left(h_{t}^{2}-\frac{1}{6} g_{1}^{2} c_{2 \beta}\right)}{m_{\tilde{Q}}^{2}}+\frac{1}{9} \frac{\left(h_{t}^{2}+\frac{1}{3} g_{1}^{2} c_{2 \beta}\right)}{m_{\tilde{t}_{R}}^{2}}+\frac{1}{36} \frac{\left(h_{t}^{2}-\frac{1}{6} g_{1}^{2} c_{2 \beta}\right)}{m_{\tilde{b}_{R}}^{2}}\right. \\
& \left.-\frac{19}{360} \frac{h_{t}^{2} X_{t}^{2}}{m_{\tilde{Q}}^{2} m_{\tilde{t}_{R}}^{2}}-\frac{1}{90} \frac{h_{b}^{2} X_{b}^{2}}{m_{\tilde{Q}}^{2} m_{\tilde{b}_{R}}^{2}}\right), \\
\bar{c}_{W W}= & \frac{m_{W}^{2}}{(4 \pi)^{2}}\left(\frac{1}{16} \frac{\left(h_{t}^{2}-\frac{1}{6} g_{1}^{2} c_{2 \beta}\right)}{m_{\tilde{Q}}^{2}}-\frac{1}{40} \frac{h_{t}^{2} X_{t}^{2}}{m_{\tilde{Q}}^{2} m_{\tilde{t}_{R}}^{2}}-\frac{1}{40} \frac{h_{b}^{2} X_{b}^{2}}{m_{\tilde{Q}}^{2} m_{\tilde{b}_{R}}^{2}}\right), \\
\bar{c}_{W B}= & \frac{m_{W}^{2}}{(4 \pi)^{2}}\left(-\frac{1}{48} \frac{\left(2 h_{t}^{2}+g_{2}^{2} c_{2 \beta}\right)}{m_{\tilde{Q}}^{2}}+\frac{1}{30} \frac{h_{t}^{2} X_{t}^{2}}{m_{\tilde{Q}}^{2} m_{\tilde{t}_{R}}^{2}}+\frac{1}{120} \frac{h_{b}^{2} X_{b}^{2}}{m_{\tilde{Q}}^{2} m_{\tilde{b}_{R}}^{2}}\right) .
\end{aligned}
$$

In the basis used in [18], the operators $\mathcal{O}_{W W}$ and $\mathcal{O}_{W B}$ are eliminated and constraints are placed on $\mathcal{O}_{\gamma} \equiv \mathcal{O}_{B B}$. The coefficients are related by $\bar{c}_{\gamma}=\bar{c}_{B B}+\bar{c}_{W W}-\bar{c}_{W B}$.

To summarise, one may calculate $\bar{c}_{g}$ and $\bar{c}_{\gamma}$ from integrating out a heavy complex scalar $\Phi$ in an arbitrary UV model by substituting the SM field matrix, $U(x)$, and field strength matrix, $G_{\mu \nu}$, into the universal one-loop effective Lagrangian of eq. (2.4). The computation of one-loop Wilson coefficients is thus reduced to evaluating the trace of a few matrices. These universal results are extendable to all dimension- 6 operators and apply also when integrating out heavy fermions and massive or massless gauge bosons [73, 84].

\section{Feynman diagram calculations and comparison}

To estimate quantitatively the validity of the dimension-6 EFT we compare the coefficients obtained above with results from an exact one-loop calculation in the MSSM. This is achieved by calculating the Feynman diagrams in figure 1 then matching the $h \rightarrow g g$ and $h \rightarrow \gamma \gamma$ amplitudes in the EFT with the equivalent MSSM amplitude. In the EFT 
the operators $\mathcal{O}_{g}$ and $\mathcal{O}_{\gamma}$ can be expanded after electroweak symmetry breaking (EWSB) around the vacuum expectation value $v \sim 174 \mathrm{GeV}$ in order to get the Lagrangian

$$
\mathcal{L}_{h V V}=g_{3}^{2} \sqrt{2} v \frac{\bar{c}_{g}}{m_{W}^{2}} h G_{\mu \nu}^{a} G^{a, \mu \nu}+g_{1}^{2} \sqrt{2} v \frac{\bar{c}_{\gamma}}{m_{W}^{2}} h B_{\mu \nu} B^{\mu \nu},
$$

corresponding to the following Feynman rules for the $h g g$ and $h \gamma \gamma$ vertices:

$$
\begin{aligned}
& i V_{h g g}^{\mu \nu}\left(p_{2}, p_{3}\right)=-4 i g_{3}^{2} \sqrt{2} v \frac{\bar{c}_{g}}{m_{W}^{2}}\left(p_{2} p_{3} g^{\mu \nu}-p_{2}^{\nu} p_{3}^{\mu}\right), \\
& i V_{h \gamma \gamma}^{\mu \nu}\left(p_{2}, p_{3}\right)=-4 i e^{2} \sqrt{2} v \frac{\bar{c}_{\gamma}}{m_{W}^{2}}\left(p_{2} p_{3} g^{\mu \nu}-p_{2}^{\nu} p_{3}^{\mu}\right) .
\end{aligned}
$$

Thus the $h \rightarrow g g$ and $h \rightarrow \gamma \gamma$ amplitudes for on-shell external particles are

$$
\begin{aligned}
& \mathcal{A}_{E F T}^{h g g}=-16 g_{s}^{2} \sqrt{2} v \frac{\bar{c}_{g}}{m_{W}^{2}}\left(\xi_{2}^{*} \cdot \xi_{3}^{*} M_{h}^{2}-2\left(\xi_{2}^{*} \cdot p_{1}\right)\left(\xi_{3}^{*} \cdot p_{1}\right)\right), \\
& \mathcal{A}_{E F T}^{h \gamma \gamma}=-2 g_{1}^{2} \cos ^{2} \theta_{W} \sqrt{2} v \frac{\bar{c}_{\gamma}}{m_{W}^{2}}\left(\xi_{2}^{*} \cdot \xi_{3}^{*} M_{h}^{2}-2\left(\xi_{2}^{*} \cdot p_{1}\right)\left(\xi_{3}^{*} \cdot p_{1}\right)\right),
\end{aligned}
$$

where the $\xi_{i}$ are the polarization vectors of the gauge bosons.

We computed the one-loop diagrams in figure 1 in the MSSM and checked our results using the FeynArts package [89]. The CP-even Higgs bosons are rotated to their physical basis by a mixing angle $\alpha$ which we set to be $\alpha=\beta-\pi / 2$ corresponding to the decoupling limit when the pseudo-scalar Higgs mass is much heavier than the mass of the $\mathrm{Z}$ gauge boson, as indicated by the experimental data $[86,87]$ and appropriate to our scenario of light stops. ${ }^{8}$

When comparing the EFT and MSSM amplitudes we may choose the momenta of the external particles to be on-shell for convenience. The result of this procedure for the $h \rightarrow g g$ amplitude yields the same expression as (3.1) with the replacement $\bar{c}_{g} \rightarrow \bar{c}_{g}^{\mathrm{MSSM}}$, where

$$
\bar{c}_{g}^{\mathrm{MSSM}}=\left(\bar{c}_{g}^{\mathrm{MSSM}}\right)^{\tilde{t}}+\left(\bar{c}_{g}^{\mathrm{MSSM}}\right)^{\tilde{b}},
$$

where the part due to stops is given by

$$
\begin{aligned}
\left(\bar{c}_{g}^{\mathrm{MSSM}}\right)^{\tilde{t}}=\frac{m_{W}^{2}}{6(4 \pi)^{2}} \frac{N_{g}^{\tilde{t}}}{D_{g}^{\tilde{t}}} & \\
N_{g}^{\tilde{t}}= & \frac{c_{2 \beta} g_{1}^{2}}{s_{W}^{2}}\left[v^{2} c_{2 \beta} g_{1}^{2}\left(2 c_{2 W}+1\right)+3\left(3 v^{2} h_{t}^{2}+2\left(m_{\tilde{t}_{R}}^{2}-m_{\tilde{Q}}^{2}\right) c_{2 W}+2 m_{\tilde{Q}}^{2}+m_{\tilde{t}_{R}}^{2}\right)\right] \\
& +36 h_{t}^{2}\left(v^{2} h_{t}^{2}+m_{\tilde{Q}}^{2}+m_{\tilde{t}_{R}}^{2}-X_{t}^{2}\right) \\
D_{g}^{\tilde{t}}= & \frac{v^{2} c_{2 \beta} g_{1}^{2}}{s_{W}^{2}}\left[v^{2} c_{2 \beta} g_{1}^{2}\left(2 c_{2 W}+1\right)+3\left(3 v^{2} h_{t}^{2}+4\left(m_{\tilde{t}_{R}}^{2}-m_{\tilde{Q}}^{2}\right) c_{2 W}+4 m_{\tilde{Q}}^{2}+2 m_{\tilde{t}_{R}}^{2}\right)\right] \\
& +36\left(v^{2} h_{t}^{2}+2 m_{\tilde{Q}}^{2}\right)\left(v^{2} h_{t}^{2}+2 m_{\tilde{t}_{R}}^{2}\right)-72 v^{2} h_{t}^{2} X_{t}^{2},
\end{aligned}
$$

${ }^{8}$ The case of relatively heavy stops has been demonstrated to be described in a very compact and convenient way, depending only on the two parameters $\tan \beta$ and the pseudo-scalar Higgs mass, when the observed Higgs mass is taken into account [86, 87]. 
and the sbottom contribution reads,

$$
\left(\bar{c}_{g}^{\mathrm{MSSM}}\right)^{\tilde{b}}=\frac{m_{W}^{2}}{6(4 \pi)^{2}} \frac{c_{2 \beta} g_{1}^{2}\left\{6\left[\left(m_{\tilde{b}_{R}}^{2}-m_{\tilde{Q}}^{2}\right) c_{2 W}+m_{\tilde{Q}}^{2}+2 m_{\tilde{b}_{R}}^{2}\right]-v^{2} c_{2 \beta} g_{1}^{2}\left(c_{2 W}+2\right)\right\}}{\left(12 m_{\tilde{b}_{R}}^{2}-v^{2} c_{2 \beta} g_{1}^{2}\right)\left[v^{2} c_{2 \beta} g_{1}^{2}\left(c_{2 W}+2\right)-24 m_{\tilde{Q}}^{2} s_{W}^{2}\right]} .
$$

For $\bar{c}_{\gamma}$ we simply have

$$
\bar{c}_{\gamma}^{\mathrm{MSSM}}=\frac{8}{3}\left(\bar{c}_{g}^{\mathrm{MSSM}}\right)^{\tilde{t}}+\frac{3}{2}\left(\bar{c}_{g}^{\mathrm{MSSM}}\right)^{\tilde{b}} .
$$

In the limit $v \rightarrow 0$ we obtain the same expressions as $\bar{c}_{g}$ and $\bar{c}_{\gamma}$ in (2.5) and (2.8), respectively. Since $\bar{c}_{g}$ and $\bar{c}_{\gamma}$ correspond to a truncation of the full theory at the dimension- 6 level, they contain only the leading-order terms in an expansion in inverse powers of the stop mass, whereas the MSSM result is exact and include higher-order terms in $v / m_{\tilde{t}, \tilde{b}}$ that would be generated by higher-dimensional operators in the EFT approach. Therefore, we expect the discrepancy between the two approaches to scale with the ratio $v / m_{\tilde{t}, \tilde{b}}$ for $m_{\tilde{t}, \tilde{b}}$, and the differences between the EFT and exact MSSM results gives insight into the potential importance of such higher-dimensional operators. We note that a large value of $X_{t}$ in terms like $v^{2} m_{W}^{2} X_{t}^{2} / m_{\tilde{t}}^{6}$ could potentially affect the validity of the EFT even for large stop masses, but the positivity of the lightest physical mass eigenvalue imposes an upper limit $X_{t} \simeq m_{\tilde{t}}^{2} / m_{t}$.

The physical mass eigenstates are obtained by diagonalizing the squark mass matrices [85]

$$
\mathcal{M}_{\tilde{q}}^{2}=\left(\begin{array}{cc}
m_{q}^{2}+m_{L L}^{2} & m_{q} X_{q} \\
m_{q} X_{q} & m_{q}^{2}+m_{R R}^{2}
\end{array}\right)
$$

with the various entries defined by

$$
\begin{aligned}
m_{L L}^{2} & =m_{\tilde{Q}}^{2}+\left(I_{q}^{3 L}-Q_{q} s_{W}^{2}\right) M_{Z}^{2} c_{2 \beta}, \\
m_{R R}^{2} & =m_{\tilde{q}_{R}}^{2}+Q_{q} s_{W}^{2} M_{Z}^{2} c_{2 \beta} \\
X_{q} & =A_{q}-\mu(\tan \beta)^{-2 I_{q}^{3 L}} .
\end{aligned}
$$

$Q_{q}$ and $I_{q}^{3 L}$ is the electromagnetic charge and the weak doublet isospin respectively. After rotating the $2 \times 2$ matrices by an angle $\theta_{q}$, which transforms the interaction eigenstates $\tilde{q}_{L}$ and $\tilde{q}_{R}$ into the mass eigenstates $\tilde{q}_{1}$ and $\tilde{q}_{2}$, the mixing angle and physical squark masses are given by

$$
\begin{aligned}
s_{2 \theta_{q}} & =\frac{2 m_{q} X_{q}}{m_{\tilde{q}_{1}}^{2}-m_{\tilde{q}_{2}}^{2}}, \quad c_{2 \theta_{q}}=\frac{m_{L L}^{2}-m_{R R}^{2}}{m_{\tilde{q}_{1}}^{2}-m_{\tilde{q}_{2}}^{2}} \\
m_{\tilde{q}_{1,2}}^{2} & =m_{q}^{2}+\frac{1}{2}\left[m_{L L}^{2}+m_{R R}^{2} \mp \sqrt{\left(m_{L L}^{2}-m_{R R}^{2}\right)^{2}+4 m_{q}^{2} X_{q}^{2}}\right] .
\end{aligned}
$$

We see that in the stop sector the mixing is strong for large values of the parameter $X_{t}=A_{t}-\mu \cot \beta$, which generates a large mass splitting between the two physical mass eigenstates and makes $\tilde{q}_{1}$ much lighter than the other sparticle $\tilde{q}_{2}$. 

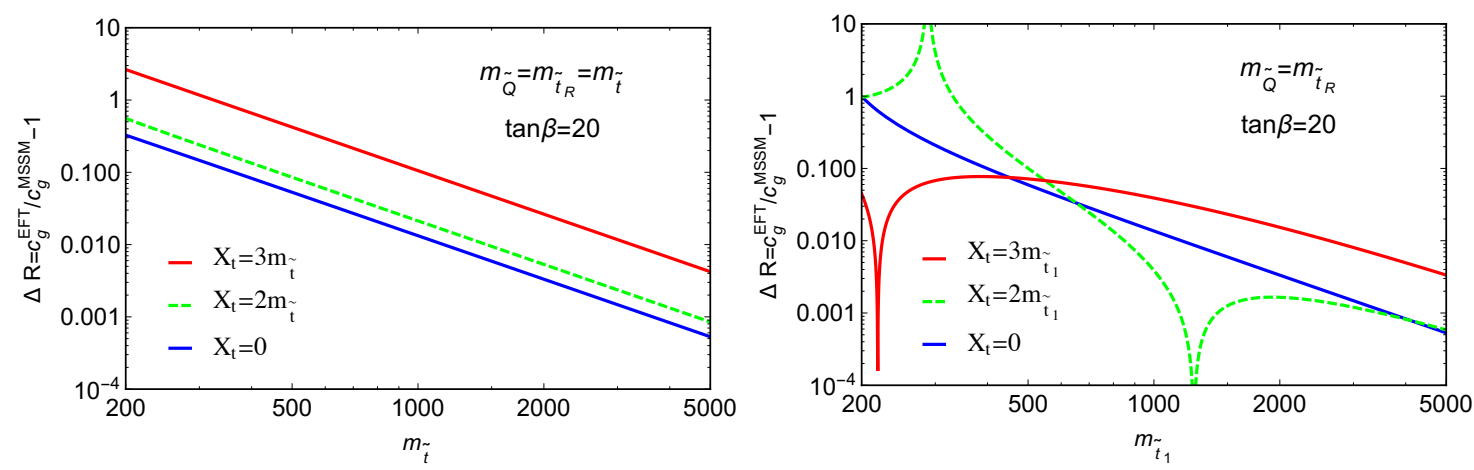

Figure 2. Values of $\Delta R$, defined in (3.11), in the degenerate case $m_{\tilde{Q}}=m_{\tilde{t}_{R}} \equiv m_{\tilde{t}}$ for $\tan \beta=20$ and the indicated values of $X_{t}$, as a function of $m_{\tilde{t}}$ (left panel), and as functions of $m_{\tilde{t}_{1}}$ (right panel).

We now compare the values of the $\bar{c}_{g}$ coefficients calculated in the MSSM and the EFT: ${ }^{9}$

$$
\Delta R \equiv \frac{\bar{c}_{g}^{\mathrm{EFT}}}{\bar{c}_{g}^{\mathrm{MSSM}}}-1
$$

Figure 2 displays values of $\Delta R$ for the degenerate case $m_{\tilde{Q}}=m_{\tilde{t}_{R}} \equiv m_{\tilde{t}}$, three different values of $X_{t}$ and the representative choice $\tan \beta=20$. In the left panel we plot $\Delta R$ as functions of $m_{\tilde{t}}$, and the right panel shows $\Delta R$ as functions of the lighter stop mass, $m_{\tilde{t}_{1}}$. We see that in both cases $\Delta R \lesssim 0.1$ for $m_{\tilde{t}}\left(m_{\tilde{t}_{1}}\right) \gtrsim 500 \mathrm{GeV}$, with a couple of exceptions. One is for the relatively large value $X_{t}=3 m_{\tilde{t}}$ in the left panel, for which $\Delta R \gtrsim 0.1$ for $m_{\tilde{t}} \lesssim 1000 \mathrm{GeV}$, and the other is for $X_{t}=2 m_{\tilde{t}_{1}}$ and $m_{\tilde{t}_{1}} \sim 290 \mathrm{GeV}$ in the right panel, which is due to a node in $\bar{c}_{g}^{\mathrm{MSSM}}$. These results serve as a warning that, although the EFT approach is in general quite reliable for stop mass parameters $\gtrsim 500 \mathrm{GeV}$, care should always be exercised for masses $\lesssim 1000 \mathrm{GeV}$.

A similar message is conveyed by figure 3 , which uses colour-coding to display values of the differences $\left|\bar{c}_{g}^{\mathrm{EFT}}-\bar{c}_{g}^{\mathrm{MSSM}}\right|$ (left panel) and $\left|\bar{c}_{\gamma}^{\mathrm{EFT}}-\bar{c}_{\gamma}^{\mathrm{MSSM}}\right|$ (right panel) in $\left(X_{t} / m_{\tilde{t}}, m_{\tilde{t}}\right)$ planes for the degenerate case $m_{\tilde{Q}}=m_{\tilde{t}_{R}} \equiv m_{\tilde{t}}$ with $\tan \beta=20$. Also shown are contours of $m_{\tilde{t}_{1}}=200 \mathrm{GeV}$ (red), $500 \mathrm{GeV}$ (green) and $1 \mathrm{TeV}$ (yellow) and regions where the $\tilde{t}_{1}$ becomes tachyonic (shaded grey). We see that the differences are generally $<2.5 \times 10^{-6}$ for $\left|\bar{c}_{g}^{\mathrm{EFT}}-\bar{c}_{g}^{\mathrm{MSSM}}\right|$ and $<10^{-5}$ for $\left|\bar{c}_{\gamma}^{\mathrm{EFT}}-\bar{c}_{\gamma}^{\mathrm{MSSM}}\right|$ when $m_{\tilde{t}_{1}}>500 \mathrm{GeV}$, even for large values of $X_{t}$, but that much larger differences are possible for $m_{\tilde{t}_{1}}<200 \mathrm{GeV}$, even for small values of $X_{t}$.

\section{Constraints on light stops from a global fit}

We now discuss the constraints on the lighter stop mass that are imposed by the current experimental constraints on the coefficients $\bar{c}_{g}$ and $\bar{c}_{\gamma}$, comparing them with the constraints imposed by electroweak precision observables via the oblique parameters $S$ and $T$ [104-107],

\footnotetext{
${ }^{9}$ We omit RGE effects that mix the coefficients in the running [90-97], as they would be higher-order corrections beyond the one-loop level of our analysis.
} 

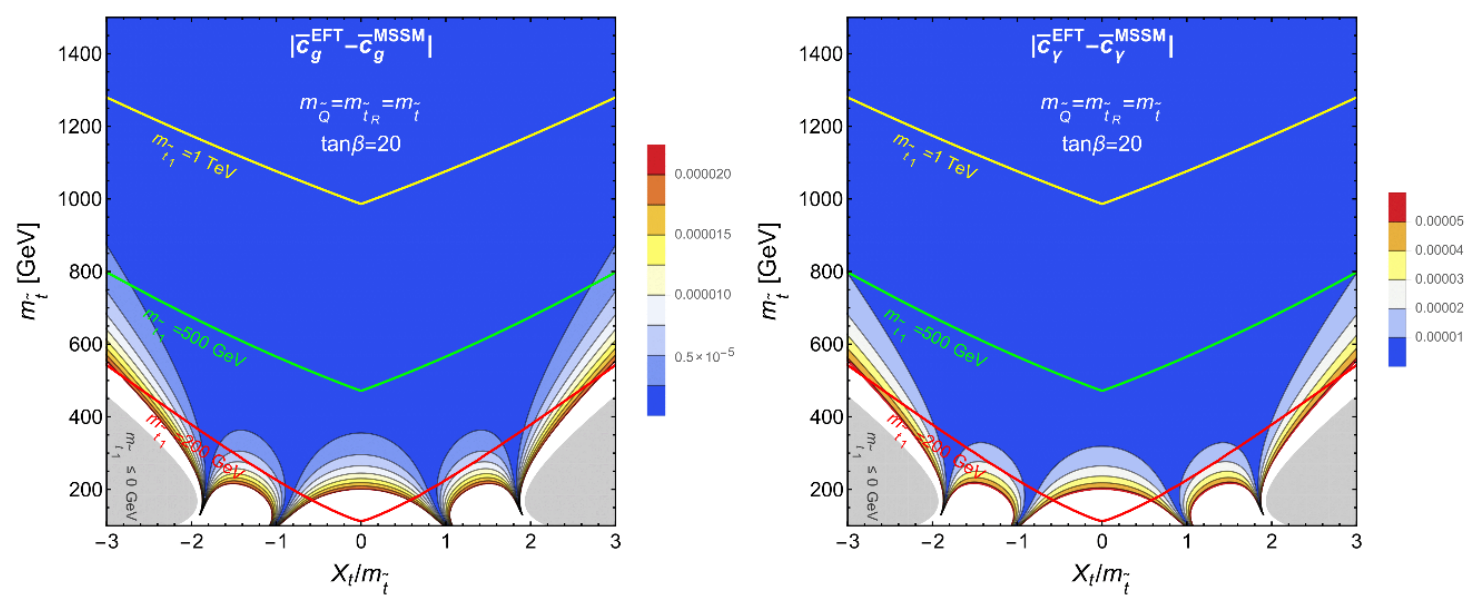

Figure 3. Contours of the differences $\left|\bar{c}_{g}^{\mathrm{EFT}}-\bar{c}_{g}^{\mathrm{MSSM}}\right|$ (left panel) and $\left|\bar{c}_{\gamma}^{\mathrm{EFT}}-\bar{c}_{\gamma}^{\mathrm{MSSM}}\right|$ (right panel) in $\left(X_{t} / m_{\tilde{t}}, m_{\tilde{t}}\right)$ planes for the degenerate case $m_{\tilde{Q}}=m_{\tilde{t}_{R}} \equiv m_{\tilde{t}}$ with $\tan \beta=20$. Also shown are contours of $m_{\tilde{t}_{1}}=200 \mathrm{GeV}, 500 \mathrm{GeV}$ and $1 \mathrm{TeV}$ and regions where the $\tilde{t}_{1}$ becomes tachyonic.

as well as the ranges favoured by measurements of the Higgs mass $M_{h}$ and direct searches at the LHC. We note that the $S$ and $T$ parameters are related to the dimension- 6 operator coefficients $\bar{c}_{W}, \bar{c}_{B}$ and $\bar{c}_{T}$, as defined in the basis of [18], ${ }^{10}$ through

$$
\begin{aligned}
& S=\frac{4 \sin ^{2} \theta_{W}}{\alpha\left(m_{Z}\right)}\left(\bar{c}_{W}+\bar{c}_{B}\right) \approx 119\left(\bar{c}_{W}+\bar{c}_{B}\right), \\
& T=\frac{1}{\alpha\left(m_{Z}\right)} \bar{c}_{T} \approx 129 \bar{c}_{T} .
\end{aligned}
$$

We shall quote the electroweak precision constraints on $\bar{c}_{W}+\bar{c}_{B}$ and $\bar{c}_{T}$ instead of $S$ and $T$, in keeping with the EFT approach. The stop contributions to these coefficients were given in [72, 73], and table 1 displays the current experimental constraints on $\bar{c}_{g}, \bar{c}_{\gamma}, \bar{c}_{T}$ and $\bar{c}_{W}+\bar{c}_{B}$ that we apply.

The constraints on the coefficients in the penultimate column of table 1 are taken from a recent global analysis [18] of LEP, LHC and Tevatron data on Higgs production and triple-gauge couplings. For $\bar{c}_{g}$ and $\bar{c}_{\gamma}$ we list the current 95\% CL ranges after marginalising a two-parameter fit in which both $\bar{c}_{g}$ and $\bar{c}_{\gamma}$ are allowed to vary, ${ }^{11}$ as well as considering the more restrictive ranges found when only $\bar{c}_{g}$ or $\bar{c}_{\gamma} \neq 0$ individually, with the other operator coefficients set to zero. Similar marginalized and individual 95\% CL limits on $\bar{c}_{T}$ and $\bar{c}_{W}+\bar{c}_{B}$ are displayed, where the two-parameter fit varying $\bar{c}_{T}$ and $\bar{c}_{W}+\bar{c}_{B}$ simultaneously is equivalent to the $S, T$ ellipse, as reproduced in [18]. We note that the stop contributions to the coefficients of the other relevant operators are far smaller than the ranges of these coefficients that were found in the global fit. This indicates that one is justified in setting

\footnotetext{
${ }^{10}$ In other bases $\bar{c}_{W}$ and $\bar{c}_{B}$ may be eliminated in favour of $\bar{c}_{W B}$.

${ }^{11}$ In any specific model there may be model-dependent correlations between operator coefficients. In the case with only light stops and nothing else one expects the relation between $\bar{c}_{g}$ and $\bar{c}_{\gamma}$ shown in (3.4) to hold, as studied in [88]. Here we use the more conservative marginalized ranges shown in the middle and right panels of figure 4 , thereby allowing for additional loop contributions to $\bar{c}_{g}$ or $\bar{c}_{\gamma}$.
} 


\begin{tabular}{|c|c|c|c|c|}
\hline Coeff. & \multicolumn{2}{|c|}{ Experimental constraints } & $95 \%$ CL limit & deg. $m_{\tilde{t}_{1}}$ \\
\hline $\bar{c}_{g}$ & LHC & $\begin{array}{c}\text { marginalized } \\
\text { individual }\end{array}$ & $\begin{array}{l}{[-4.5,2.2] \times 10^{-5}} \\
{[-3.0,2.5] \times 10^{-5}}\end{array}$ & $\begin{array}{l}\sim 410 \mathrm{GeV} \\
\sim 390 \mathrm{GeV}\end{array}$ \\
\hline $\bar{c}_{\gamma}$ & LHC & $\begin{array}{c}\text { marginalized } \\
\text { individual }\end{array}$ & $\begin{array}{l}{[-6.5,2.7] \times 10^{-4}} \\
{[-4.0,2.3] \times 10^{-4}}\end{array}$ & $\begin{array}{l}\sim 215 \mathrm{GeV} \\
\sim 230 \mathrm{GeV}\end{array}$ \\
\hline $\bar{c}_{T}$ & LEP & $\begin{array}{c}\text { marginalized } \\
\text { individual }\end{array}$ & $\begin{array}{c}{[-10,10] \times 10^{-4}} \\
{[-5,5] \times 10^{-4}}\end{array}$ & $\begin{array}{l}\sim 290 \mathrm{GeV} \\
\sim 380 \mathrm{GeV}\end{array}$ \\
\hline $\bar{c}_{W}+\bar{c}_{B}$ & LEP & $\begin{array}{c}\text { marginalized } \\
\text { individual }\end{array}$ & $\begin{array}{l}{[-7,7] \times 10^{-4}} \\
{[-5,5] \times 10^{-4}}\end{array}$ & $\begin{array}{l}\sim 185 \mathrm{GeV} \\
\sim 195 \mathrm{GeV}\end{array}$ \\
\hline
\end{tabular}

Table 1. List of the experimental 95\% CL bounds on coefficients used in setting current limits on stops, which are taken from [18]. The marginalized LHC limits are for a two-parameter fit allowing $\bar{c}_{g}$ and $\bar{c}_{\gamma}$ to vary, and the marginalized LEP limits are for a two-parameter fit of $\bar{c}_{T}$ and $\bar{c}_{W}+\bar{c}_{B}$. The corresponding lightest stop mass limits shown are for degenerate soft-supersymmetry breaking masses $m_{\tilde{Q}}=m_{\tilde{t}_{R}}=m_{\tilde{t}}$ with $X_{t}=0$.
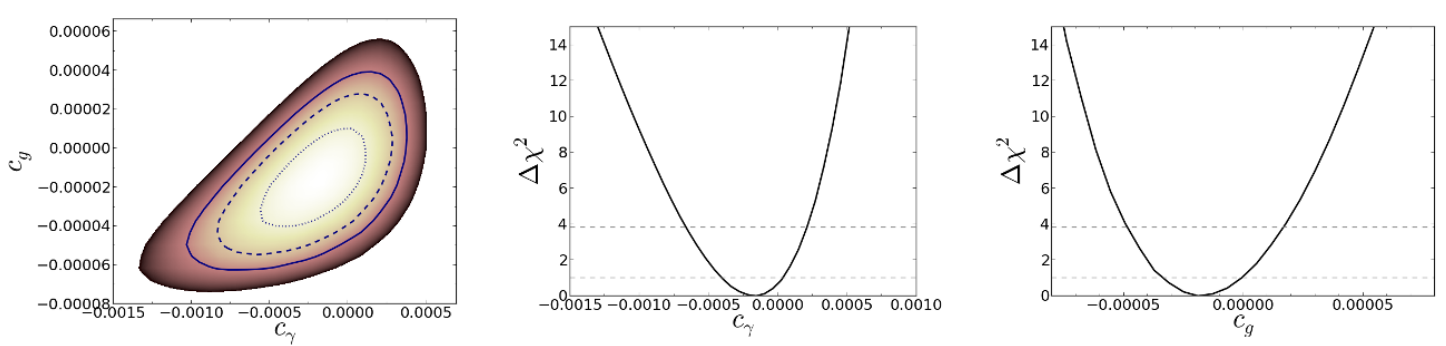

Figure 4. Results based on the global fit in [18], varying $\bar{c}_{g}$ and $\bar{c}_{\gamma}$ simultaneously but setting to zero the coefficients of the other dimension- 6 operators contributing to the Higgs sector. The dotted, dashed and solid contours on the left denote the allowed $68 \%, 95 \%$ and $99 \%$ CL regions respectively. The middle and right figures show the marginalized $\chi^{2}$ functions for $\bar{c}_{\gamma}$ and $\bar{c}_{g}$ respectively.

these other operator coefficients to zero when considering bounds on the stop sector, if one assumes that there are no important contributions from other possible new physics.

\subsection{Degenerate stop masses}

Figure 5 displays the current constraints in the case of degenerate soft masses $m_{\tilde{Q}}=$ $m_{\tilde{t}_{R}} \equiv m_{\tilde{t}}$ with decoupled sbottoms, in the upper panels for $m_{\tilde{t}}$ as functions of $X_{t} / m_{\tilde{t}}$ and in the lower panels for $m_{\tilde{t}_{2}}$ as functions of $m_{\tilde{t}_{1}}$, in both cases for $\tan \beta=20$. The left panels show the stop constraints from the current marginalized $95 \%$ bounds on $\bar{c}_{g}$ (red lines) and $\bar{c}_{\gamma}$ (blue lines), and the right panels show the corresponding bounds from the current marginalized $95 \%$ bounds. The solid (dashed) lines are obtained from an exact one-loop MSSM analysis and the EFT approach, respectively. The purple lines show the individual bound from $\bar{c}_{T}$ in the EFT approach. The bounds from $\bar{c}_{W}+\bar{c}_{B}$ corresponding to the $S$ parameter are negligible and omitted here. The grey shaded regions are excluded 

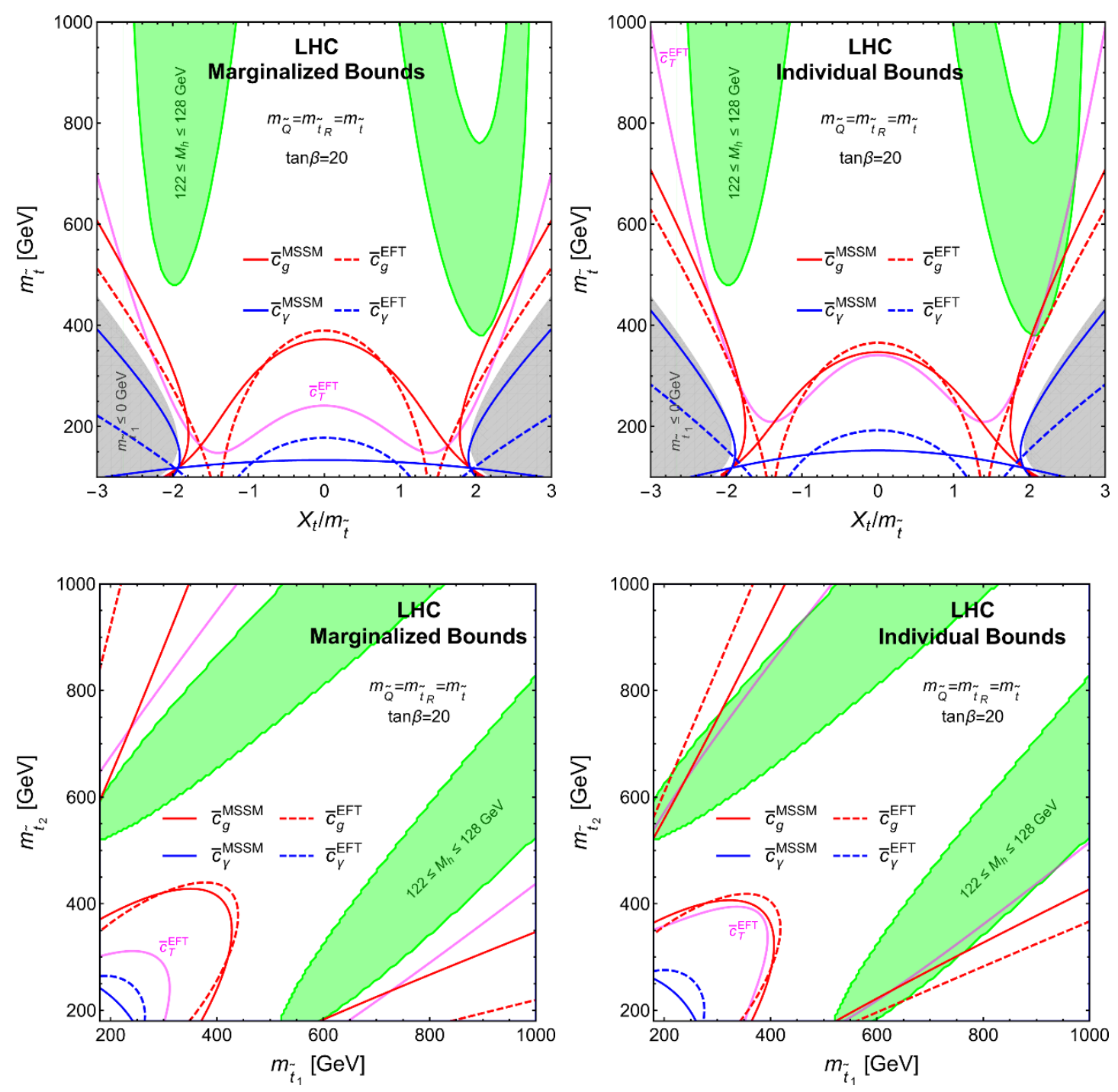

Figure 5. Compilation of the constraints in (upper panels) the $\left(X_{t} / m_{\tilde{t}_{1}}, m_{\tilde{t}}\right)$ plane and (lower panels) the $\left(m_{\tilde{t}}, m_{\tilde{t}_{2}}\right)$ plane from (left panels) the marginalized bounds on $\bar{c}_{g}$ (red lines) and $\bar{c}_{\gamma}$ (blue lines), and from (right panels) the individual bounds on $\bar{c}_{g}$ and $\bar{c}_{\gamma}$. Also shown are the EFT bounds on $\bar{c}_{T}$ (purple lines), the constraint that the lighter stop should not be tachyonic (grey shading) and the region where $M_{h} \in(122,128) \mathrm{GeV}$ according to a FeynHiggs 2.10.3 [98-102] calculation assuming no other significant contributions from outside the stop sector (green shading).

because the lighter stop becomes tachyonic, and the green shaded regions correspond to $122 \mathrm{GeV}<M_{h}<128 \mathrm{GeV}$, as calculated using FeynHiggs 2.10.3 [98-102], allowing for a theoretical uncertainty of $\pm 3 \mathrm{GeV}$ and assuming that there are no other important MSSM contributions to $M_{h}$.

We see in the upper panels of figure 5 that the $\bar{c}_{g}$ constraints on $m_{\tilde{t}_{1}}$ are generally the strongest, except for large $\left|X_{t} / m_{\tilde{t}}\right|$. We also observe that the MSSM and EFT evaluations give rather similar bounds on $m_{\tilde{t}_{1}}$ for $\left|X_{t} / m_{\tilde{t}}\right| \lesssim 1$ and $\gtrsim 2$. However, there are 
significant differences for $1 \lesssim\left|X_{t} / m_{\tilde{t}}\right| \lesssim 2$, due to the fact that the two evaluations have zeroes at different values of $X_{t} / m_{\tilde{t}}$. The next most sensitive constraints are those from $T$, parametrised here by the coefficient $\bar{c}_{T}$, which become competitive with the $\bar{c}_{g}$ constraints at large $\left|X_{t} / m_{\tilde{t}}\right|$, but are significantly weaker for small values of $X_{t} / m_{\tilde{t}}$. The constraints from $\bar{c}_{\gamma}$ are weaker still for all values of $X_{t} / m_{\tilde{t}}$, as might have been expected because the global fit in [18] gave constraints on $\bar{c}_{\gamma}$ that are weaker than those on $\bar{c}_{g}$. Indeed, the $\bar{c}_{\gamma}$ constraint is not significantly stronger than the constraint that the $\tilde{t}_{1}$ not be tachyonic, as shown by the grey shading in the upper panels of figure 5. We also note that the LHC measurement of $M_{h}$ favours $\left|X_{t} / m_{\tilde{t}}\right| \gtrsim 2$ and values of $m_{\tilde{t}}$ that are consistent with the EFT bounds.

These results are reflected in the lower panels of figure 5, where we present the $\left(m_{\tilde{t}_{1}}, m_{\tilde{t}_{2}}\right)$ planes with the marginalized constraints (left panel) and the individual constraints (right panel). The MSSM and EFT implementations of the $\bar{c}_{g}$ constraint give qualitatively similar results, and (except for extreme values of $m_{\tilde{t}_{1}} / m_{\tilde{t}_{2}}$ ) are generally stronger than the constraints from $\bar{c}_{T}$, which are in turn stronger than the $\bar{c}_{\gamma}$ constraint. We also note that the LHC measurement of $M_{h}$ favours moderate values of $m_{\tilde{t}_{1}} / m_{\tilde{t}_{2}}$ and values of $m_{\tilde{t}_{1}}$ or $m_{\tilde{t}_{2}} \gtrsim 520 \mathrm{GeV}$.

The limits on the lightest stop mass for degenerate soft-supersymmetry breaking masses $m_{\tilde{Q}}=m_{\tilde{t}_{R}}=m_{\tilde{t}}$ with $X_{t}=0$ are shown in the last column of table 1 .

\subsection{Non-degenerate stop masses}

We consider now cases with non-degenerate stop soft mass parameters, allowing also for the possibility that the lighter sbottom squark plays a rôle. We show in figure 6 various planes under the hypotheses $m_{\tilde{b}_{1}}=m_{\tilde{t}_{1}}$ and $\tan \beta=20$, considering several possibilities for $X_{t}$. In all panels, the constraints from the individual $95 \%$ bound on $\bar{c}_{g}$ are indicated by red lines and those from $\bar{c}_{\gamma}$ are indicated by blue lines (solid for the exact MSSM evaluation and dashed for the EFT approach), and the region allowed by the exact calculation is shaded pink.

The upper left panel is for $X_{t}=0$ : we see that in the limit $m_{\tilde{t}_{2}} \gg m_{\tilde{t}_{1}}$ the $\bar{c}_{g}$ constraint imposes $m_{\tilde{t}_{1}} \gtrsim 300 \mathrm{GeV}$, with a difference of $\sim 20 \mathrm{GeV}$ between the exact and EFT calculations. On the other hand, if $m_{\tilde{t}_{2}}=m_{\tilde{t}_{1}}$ we find $m_{\tilde{t}_{1}} \gtrsim 380 \mathrm{GeV}$, again with the EFT calculation giving a bound $\sim 20 \mathrm{GeV}$ stronger than the exact MSSM calculation. The corresponding bounds from the individual $95 \%$ constraint on $\bar{c}_{\gamma}$ are $\simeq 100 \mathrm{GeV}$ weaker. However, we note that the LHC constraint on $M_{h}$ is not respected anywhere in this plane.

Turning now to the case $X_{t}=1 \mathrm{TeV}$ shown in the upper right panel of figure 6 , we see a grey shaded band around the $m_{\tilde{t}_{1}}=m_{\tilde{t}_{2}}$ line that is disallowed by $\tilde{t}_{1}-\tilde{t}_{2}$ mixing, and other grey shaded regions where $m_{\tilde{t}_{1}} \ll m_{\tilde{t}_{2}}$ (or vice versa) and the lighter stop is tachyonic. In this case the $M_{h}$ constraint (green shaded band) can be satisfied, with small strips of the parameter space ruled out by the $\bar{c}_{g}$ constraint. The $\bar{c}_{\gamma}$ constraint is unimportant in this case.

When $X_{t}$ is increased to $3 \mathrm{TeV}$, as shown in the lower left panel of figure 6 , the diagonal band forbidden by mixing expands considerably, and the $\bar{c}_{\gamma}$ constraint disappears. In this case the $\bar{c}_{g}$ constraint would allow $\left(m_{\tilde{t}_{1}}, m_{\tilde{t}_{2}}\right) \gtrsim(400,1100) \mathrm{GeV}$ on the boundary of the 

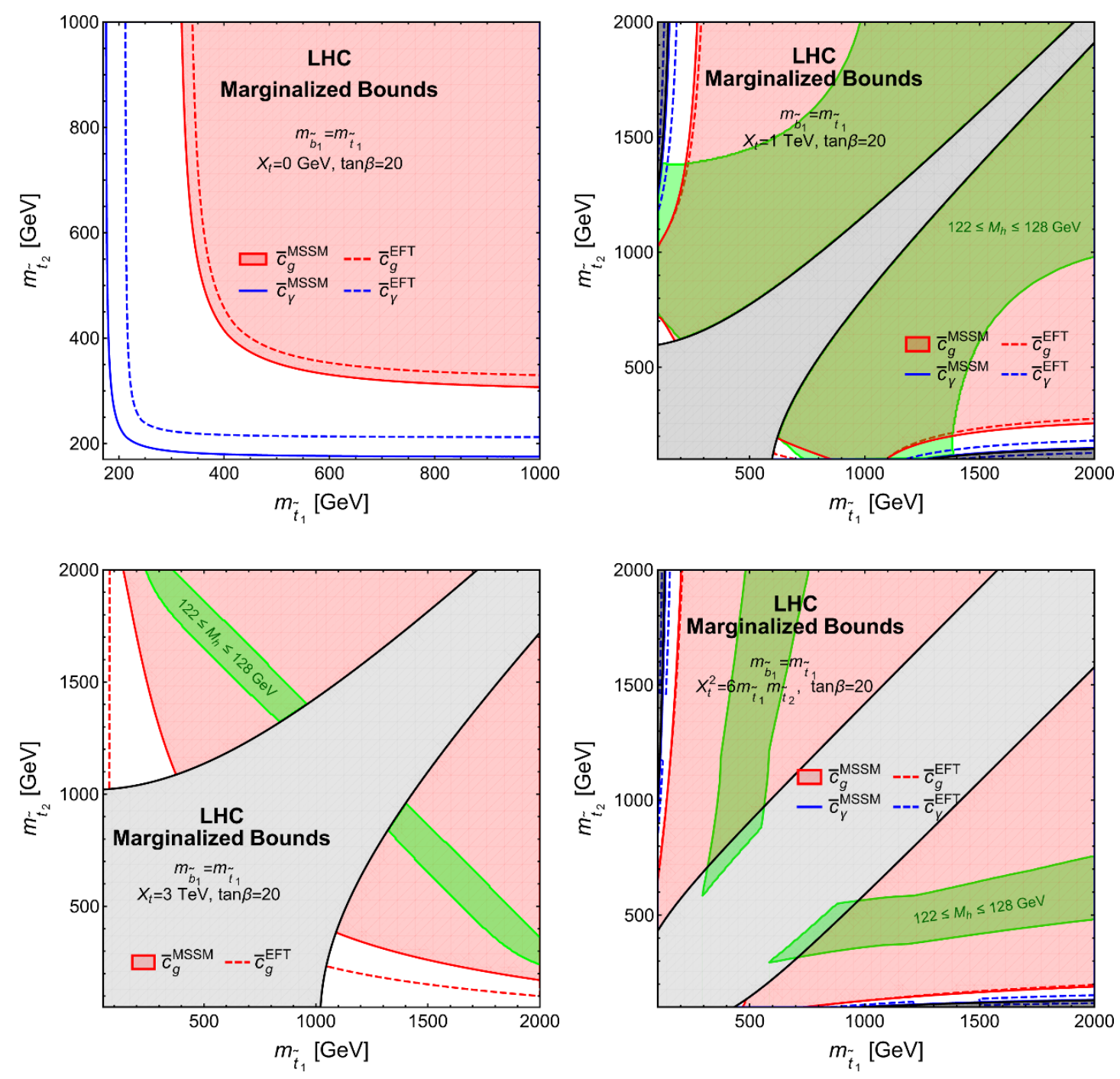

Figure 6. Compilation of the constraints in the case of non-degenerate soft mass parameters, including also sbottom squarks and assuming $m_{\tilde{b}_{1}}=m_{\tilde{t}_{1}}$ under the hypotheses $\tan \beta=20$ and $X_{t}=0$ (upper left panel), $X_{t}=1 \mathrm{TeV}$ (upper right panel), $X_{t}=3 \mathrm{TeV}$ (lower left panel) and $X_{t}=\sqrt{6 m_{\tilde{t}_{1}} m_{\tilde{t}_{2}}}$ (lower right panel). The red (blue) lines show the current individual $95 \% \mathrm{CL}$ constraints from $\bar{c}_{g}\left(\bar{c}_{\gamma}\right)$ as evaluated exactly in the MSSM (solid lines) and in the EFT approach. Additionally, the region compatible with $\bar{c}_{g}$ is shaded pink, the band compatible with $M_{h}$ is shaded green, and regions disallowed by the mixing hypothesis or the appearance of a tachyonic stop are shaded grey.

band forbidden by the mixing hypothesis, but the $M_{h}$ constraint is stronger, enforcing $\left(m_{\tilde{t}_{1}}, m_{\tilde{t}_{2}}\right) \gtrsim(800,1300) \mathrm{GeV}$ along this boundary.

Finally, we consider in the lower right panel of figure 6 the so-called maximal-mixing hypothesis $X_{t}=\sqrt{6 m_{\tilde{t}_{1}} m_{\tilde{t}_{2}}}$. In this case, almost the entire $\left(m_{\tilde{t}_{1}}, m_{\tilde{t}_{2}}\right)$ plane is allowed by the $\bar{c}_{g}$ constraint, whereas a triangular region at small $m_{\tilde{t}_{1}}$ and/or $m_{\tilde{t}_{2}}$ is forbidden by the $M_{h}$ constraint. 
It is interesting to compare the limits on $m_{\tilde{t}_{1}}$ that we find with those found in a recent global fit to the pMSSM [103] in which universal third-generation squark masses were assumed at the renormalisation scale $\sqrt{m_{\tilde{t}_{1}} m_{\tilde{t}_{2}}}$, the first- and second-generation squark masses were assumed to be equal, but allowed to differ from the third-generation mass as were the slepton masses, arbitrary non-universal gaugino masses $M_{1,2,3}$ were allowed, and the trilinear soft supersymmetry-breaking parameter $A$ was assumed to be universal but otherwise free. That analysis included LHC, dark matter and flavour constraints, as well as electroweak precision observables and Higgs measurements, and found $m_{\tilde{t}_{1}} \gtrsim$ $400 \mathrm{GeV}$. The analysis of this paper uses somewhat different assumptions and hence is not directly comparable, but it is interesting that the one-loop sensitivity of $\bar{c}_{g}$ to the stop mass parameters is quite comparable.

\section{Sensitivities of possible future precision measurements}

We saw in the previous section that the precision of current measurements does not exclude in a model-independent way most of the parameter space for a stop below the TeV scale, and barely reaches into the region required for a $125 \mathrm{GeV}$ Higgs mass in the MSSM. However, future colliders will increase significantly the precision of electroweak and Higgs measurements to the level required to challenge seriously the naturalness paradigm and test the MSSM calculations of $M_{h}$.

In this section we assess the potential improvements for constraints on a light stop possible with future $e^{+} e^{-}$colliders. As previously, we perform an analysis in the EFT framework via the corresponding bounds on the relevant dimension- 6 coefficients, and compare it with the exact one-loop MSSM calculation. As representative examples of future $e^{+} e^{-}$colliders, we focus on the ILC [110] and FCC-ee [108, 109] (formerly known as TLEP) proposals. The scenarios considered here for the ILC and FCC-ee postulate centre-of-mass energies of 250 and $240 \mathrm{GeV}$ with luminosities of $1150 \mathrm{fb}^{-1}$ and $10000 \mathrm{fb}^{-1}$, respectively.

Table 2 lists the prospective $95 \%$ CL limits obtained on $\bar{c}_{g}, \bar{c}_{\gamma}, \bar{c}_{T}$, and $\bar{c}_{W}+\bar{c}_{B}$ from a $\chi^{2}$ analysis, with the marginalized constraints on $\bar{c}_{g}$ and $\bar{c}_{\gamma}$ obtained in a two-parameter fit to just these coefficients, and similarly for $\bar{c}_{T}$ and $\bar{c}_{W}+\bar{c}_{B}$, corresponding to the $T$ and $S$ parameters respectively, as well as the constraints obtained when each operator coefficient is allowed individually to be non-zero. The target precisions on experimental errors for the electroweak precision observables $m_{W}, \Gamma_{Z}, R_{l}$ and $A_{l}$ at the ILC are given in [110], and those at FCC-ee were taken from [108, 109], and include important systematic uncertainties. The errors on the Higgs associated production cross-section times branching ratio are from [111] for the ILC and from [83] for FCC-ee. The numbers quoted in table 2 neglect theoretical uncertainties, in order to reflect the possible performances of the experiments. ${ }^{12}$ The treatment of the dimension- 6 coefficients in the observables follows a procedure similar to that of the global fit performed in [18], and we use the results of [74] to rescale the constraint from associated Higgs production.

\footnotetext{
${ }^{12}$ We also show as dashed purple lines in the FCC-ee panels the weaker constraints obtained using the estimates of theoretical uncertainties in [112], while noting that these have not been studied in detail.
} 


\begin{tabular}{|c|c|c|c|c|c|}
\hline \multirow{2}{*}{ Coeff. } & \multirow{2}{*}{\multicolumn{2}{|c|}{ Experimental constraints }} & \multirow{2}{*}{$95 \%$ CL limit } & \multicolumn{2}{|c|}{ deg. $m_{\tilde{t}_{1}}$} \\
\hline & & & & $X_{t}=0$ & $X_{t}=m_{\tilde{t}} / 2$ \\
\hline \multirow{4}{*}{$\bar{c}_{g}$} & \multirow{2}{*}{$\mathrm{ILC}_{250 \mathrm{GeV}}^{1150 \mathrm{fb}^{-1}}$} & marginalized & {$[-7.7,7.7] \times 10^{-6}$} & $\sim 675 \mathrm{GeV}$ & $\sim 520 \mathrm{GeV}$ \\
\hline & & individual & {$[-7.5,7.5] \times 10^{-6}$} & $\sim 680 \mathrm{GeV}$ & $\sim 545 \mathrm{GeV}$ \\
\hline & \multirow{2}{*}{ FCC-ee } & marginalized & {$[-3.0,3.0] \times 10^{-6}$} & $\sim 1065 \mathrm{GeV}$ & $\sim 920 \mathrm{GeV}$ \\
\hline & & individual & {$[-3.0,3.0] \times 10^{-6}$} & $\sim 1065 \mathrm{GeV}$ & $\sim 915 \mathrm{GeV}$ \\
\hline \multirow{4}{*}{$\bar{c}_{\gamma}$} & \multirow{2}{*}{$\mathrm{ILC}_{250 \mathrm{GeV}}^{1150 \mathrm{fb}^{-1}}$} & marginalized & {$[-3.4,3.4] \times 10^{-4}$} & $\sim 200 \mathrm{GeV}$ & $\sim 40 \mathrm{GeV}$ \\
\hline & & individual & {$[-3.3,3.3] \times 10^{-4}$} & $\sim 200 \mathrm{GeV}$ & $\sim 35 \mathrm{GeV}$ \\
\hline & \multirow{2}{*}{ FCC-ee } & marginalized & {$[-6.4,6.4] \times 10^{-5}$} & $\sim 385 \mathrm{GeV}$ & $\sim 250 \mathrm{GeV}$ \\
\hline & & individual & {$[-6.3,6.3] \times 10^{-5}$} & $\sim 390 \mathrm{GeV}$ & $\sim 260 \mathrm{GeV}$ \\
\hline \multirow{4}{*}{$\bar{c}_{T}$} & \multirow{2}{*}{$\mathrm{ILC}_{250 \mathrm{GeV}}^{1150 \mathrm{fb}^{-1}}$} & marginalized & {$[-3,3] \times 10^{-4}$} & $\sim 480 \mathrm{GeV}$ & $\sim 285 \mathrm{GeV}$ \\
\hline & & individual & {$[-7,7] \times 10^{-5}$} & $\sim 930 \mathrm{GeV}$ & $\sim 780 \mathrm{GeV}$ \\
\hline & \multirow{2}{*}{ FCC-ee } & marginalized & {$[-3,3] \times 10^{-5}$} & $\sim 1410 \mathrm{GeV}$ & $\sim 1285 \mathrm{GeV}$ \\
\hline & & individual & {$[-0.9,0.9] \times 10^{-5}$} & $\sim 2555 \mathrm{GeV}$ & $\sim 2460 \mathrm{GeV}$ \\
\hline \multirow{4}{*}{$\bar{c}_{W}+\bar{c}_{B}$} & \multirow{2}{*}{$\mathrm{ILC}_{250 \mathrm{GeV}}^{1150 \mathrm{fb}^{-1}}$} & marginalized & {$[-2,2] \times 10^{-4}$} & $\sim 230 \mathrm{GeV}$ & $\sim 170 \mathrm{GeV}$ \\
\hline & & individual & {$[-6,6] \times 10^{-5}$} & $\sim 340 \mathrm{GeV}$ & $\sim 470 \mathrm{GeV}$ \\
\hline & \multirow{2}{*}{ FCC-ee } & marginalized & {$[-2,2] \times 10^{-5}$} & $\sim 545 \mathrm{GeV}$ & $\sim 960 \mathrm{GeV}$ \\
\hline & & individual & {$[-0.8,0.8] \times 10^{-5}$} & $\sim 830 \mathrm{GeV}$ & $\sim 1590 \mathrm{GeV}$ \\
\hline
\end{tabular}

Table 2. List of the 95\% CL bounds on EFT operator coefficients from projected constraints on Higgs couplings and electroweak precision observables at the future $e^{+} e^{-}$colliders ILC and FCCee. The marginalized limits on $\bar{c}_{g}$ or $\bar{c}_{\gamma}\left(\bar{c}_{T}\right.$ or $\left.\bar{c}_{W}+\bar{c}_{B}\right)$ are for a two-parameter fit allowing $\bar{c}_{g}$ and $\bar{c}_{\gamma}\left(\bar{c}_{T}\right.$ and $\left.\bar{c}_{W}+\bar{c}_{B}\right)$ to vary simultaneously but setting other operator coefficients to zero. The corresponding lightest stop mass limits shown are for degenerate soft-supersymmetry breaking masses $m_{\tilde{Q}}=m_{\tilde{t}_{R}}=m_{\tilde{t}}$ with $X_{t}=0$ and $X_{t} / m_{\tilde{t}}=2$.

\subsection{Degenerate stop masses}

Contours from possible future constraints on $\bar{c}_{g}, \bar{c}_{\gamma}$ and $\bar{c}_{T}$ for the case of degenerate soft masses $m_{\tilde{Q}}=m_{\tilde{t}_{R}} \equiv m_{\tilde{t}}$ are plotted in figure 7 , using again the value $\tan \beta=20$. The upper panels show results for the ILC, the lower panels for FCC-ee, the left panels show the marginalized constraints and the right panels show the individual constraints. The grey and green shaded regions are the same as in figure 7 . We see that the marginal and individual sensitivities to $m_{\tilde{t}}$ from $\bar{c}_{g}$ and $\bar{c}_{\gamma}$ are very similar, whereas the individual sensitivity of $\bar{c}_{T}$ are much stronger, particularly at FCC-ee. We see that ILC is indirectly sensitive to $m_{\tilde{t}} \sim 600 \mathrm{GeV}$, and that FCC-ee is indirectly sensitive to stops in the $\mathrm{TeV}$ range. The measurement of the $\bar{c}_{T}$ coefficient at FCC-ee has the highest potential reach, though this will be highly dependent on future improvements in reducing theory uncertainties $[83,112]$.

The limits on the lightest stop mass for degenerate soft-supersymmetry breaking masses $m_{\tilde{Q}}=m_{\tilde{t}_{R}}=m_{\tilde{t}}$ with $X_{t}=0$ and $X_{t} / m_{\tilde{t}}=2$ are shown in the two last columns of table 2 . 

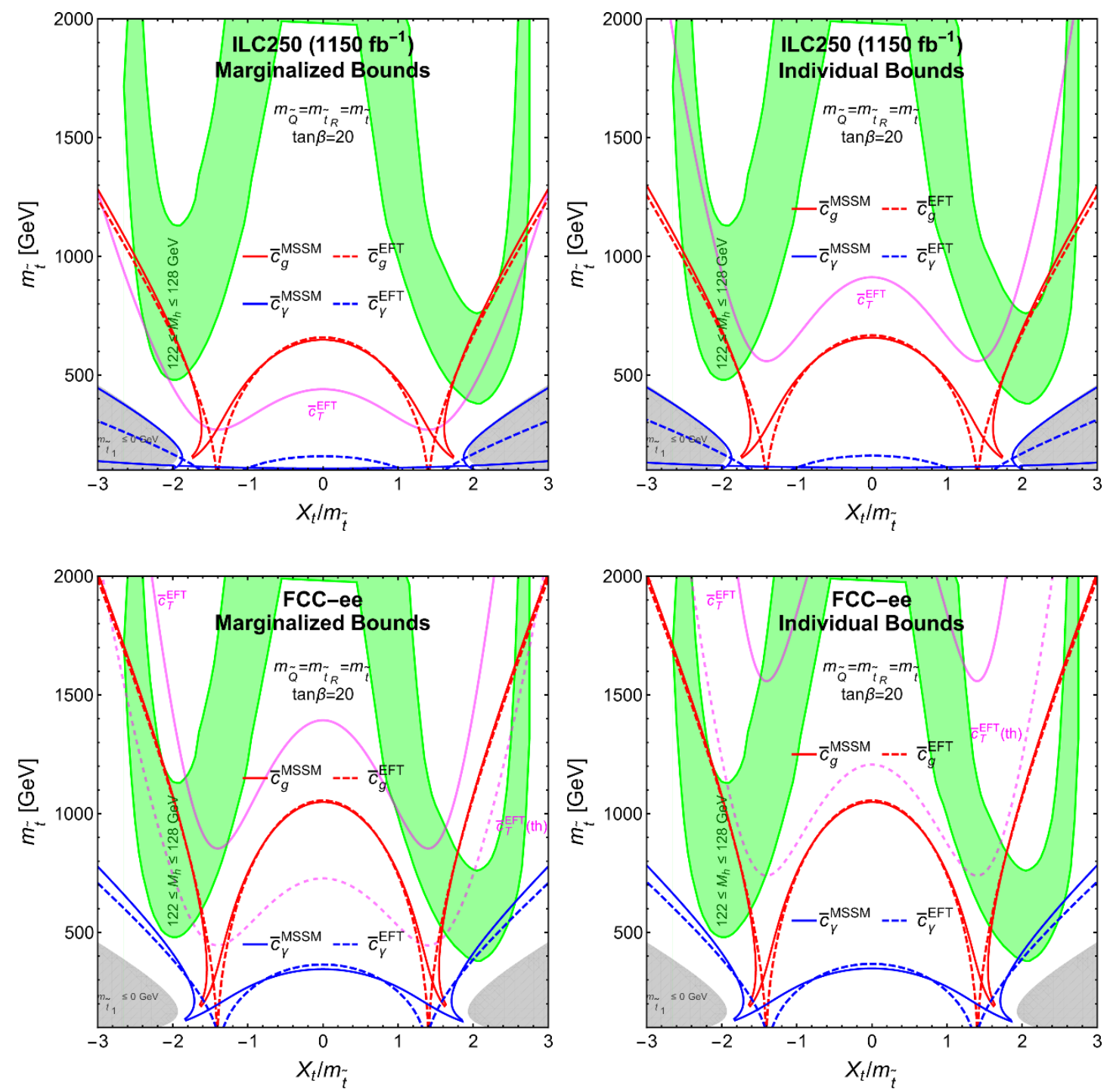

Figure 7. The $\left(X_{t} / m_{\tilde{t}}, m_{\tilde{t}}\right)$ planes, analogous to those in the upper panels of figure 5 , showing prospective marginalized bounds (left panels) and individual bounds (right panels) from the ILC [110] with $1150 \mathrm{fb}^{-1}$ of luminosity at $250 \mathrm{GeV}$ (upper panels) and from FCC-ee [108, 109] with $10^{4} \mathrm{fb}^{-1}$ of luminosity at $240 \mathrm{GeV}$ (lower panels). In the latter case, the solid purple lines are the $95 \%$ CL contours for electroweak precision measurements from FCC-ee incorporating the projected statistical and systematic experimental errors alone, and the dashed purple lines also include theory errors from [112].

\subsection{Non-degenerate stop masses}

Moving on to the non-degenerate case, the $\bar{c}_{g}$ and $\bar{c}_{\gamma} 95 \%$ CL limits for ILC and FCC-ee are plotted in the $m_{\tilde{t}_{1}}$ vs $m_{\tilde{t}_{2}}$ plane for various $X_{t}$ values in figure 8 and 9 respectively. The top left, top right, and bottom left plots correspond to $X_{t}=0,1$ and $3 \mathrm{TeV}$ respectively, while the bottom right plot is for the maximal-mixing hypothesis $X_{t}=\sqrt{6 m_{\tilde{t}_{1}} m_{\tilde{t}_{2}}}$. We see 

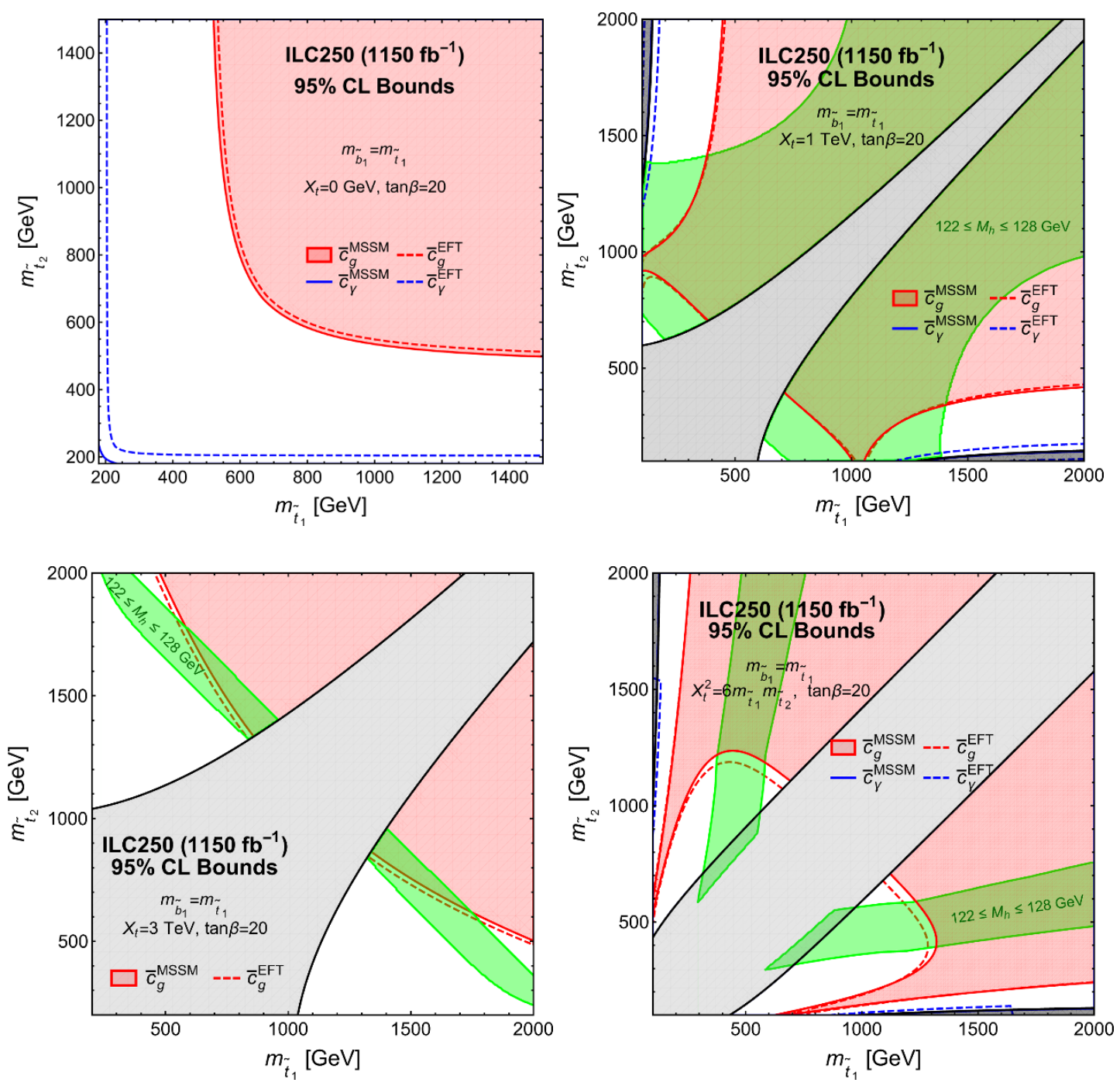

Figure 8. Compilation of projected ILC $95 \%$ CL bounds from $\bar{c}_{g}\left(\bar{c}_{\gamma}\right)$ given by red (blue) lines in the $m_{\tilde{t}_{1}}$ vs $m_{\tilde{t}_{2}}$ plane, analogous to figure 6 , with $m_{\tilde{b}_{1}}=m_{\tilde{t}_{1}}$ and $\tan \beta=20$. Values of $X_{t}=$ $0,1,3, \sqrt{6 m_{\tilde{t}_{1}} m_{\tilde{t}_{2}}} \mathrm{TeV}$ are shown clockwise from top left. The marginalized limits are displayed and the individual bounds are very similar.

that the ILC sensitivity to $\bar{c}_{g}$ begins to probe and potentially exclude parts of the green shaded region compatible with the measured $M_{h}$, while FCC-ee would push the sensitivity of $\bar{c}_{g}$ constraints into the TeV scale. In particular, it could eliminate the entire allowed $M_{h}$ region for $X_{t}=3 \mathrm{TeV}$.

\section{Conclusions and prospects}

In light of the SM-like Higgs sector and the current lack of direct evidence for additional degrees of freedom beyond the SM, the framework of the Effective SM (ESM) is gaining increasing attention as a general framework for characterising the indirect effects of possible 

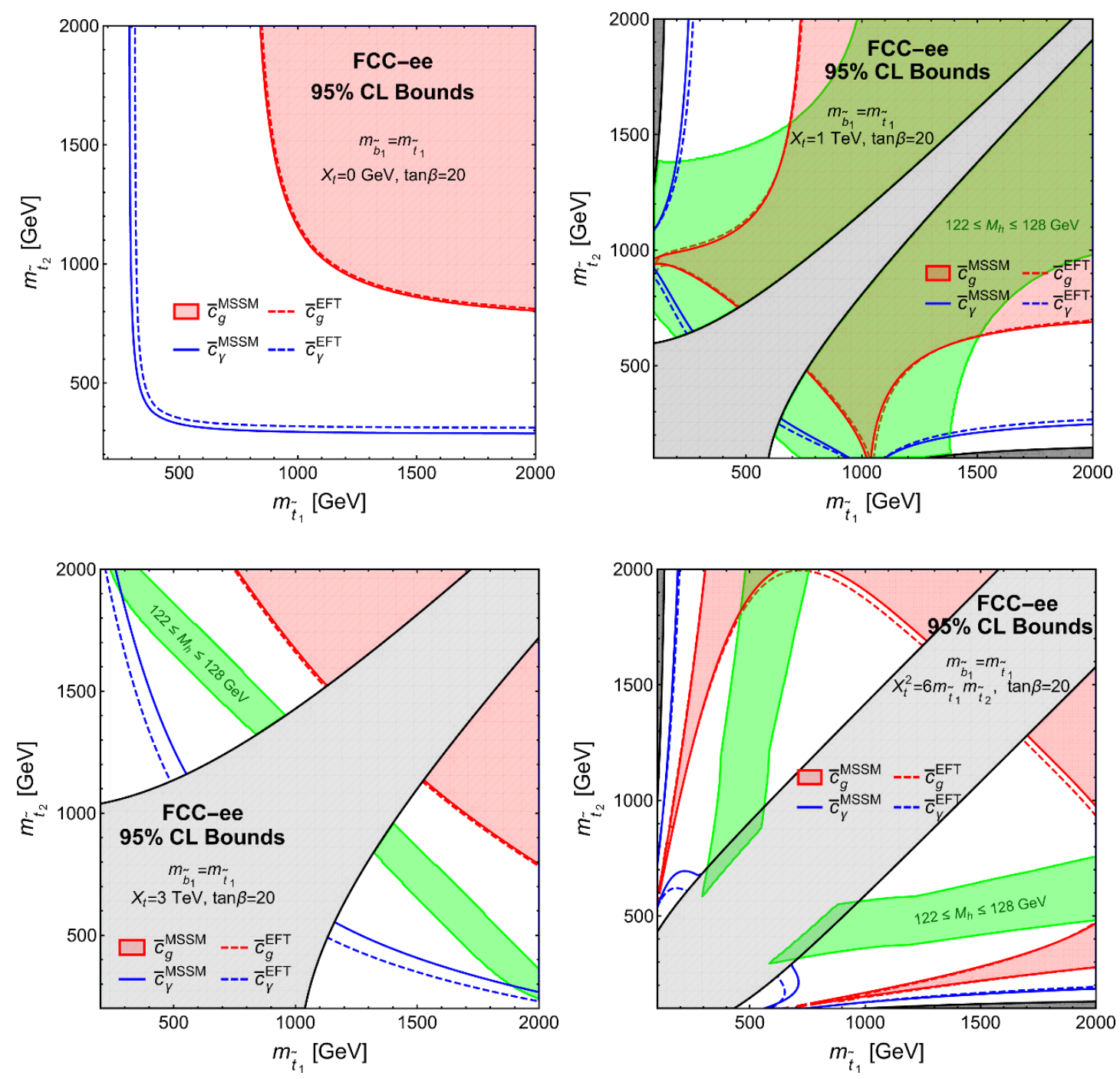

Figure 9. Compilation of projected FCC-ee $95 \%$ CL bounds from $\bar{c}_{g}\left(\bar{c}_{\gamma}\right)$ given by red (blue) lines in the $m_{\tilde{t}_{1}}$ vs $m_{\tilde{t}_{2}}$ plane, analogous to figure 6 , with $m_{\tilde{b}_{1}}=m_{\tilde{t}_{1}}$ and $\tan \beta=20$. Values of $X_{t}=0,1,3, \sqrt{6 m_{\tilde{t}_{1}} m_{\tilde{t}_{2}}} \mathrm{TeV}$ is shown clockwise from top left. The marginalized limits are displayed and the individual bounds are very similar.

new physics in a model-independent way. The ESM is simply the SM extended in the way it has always been regarded: as an effective field theory supplemented by higher-dimensional operators suppressed by the scale of new physics. The leading lepton-number-conserving effects are parametrised by dimension- 6 operators, whose coefficients are determined by matching to a UV model and constrained through their effects on experimental observables. In this paper we have illustrated all these steps in the EFT approach for light stops in the MSSM.

In particular, we employed the CDE method to compute the one-loop effective Lagrangian, showing how certain results derived previously under the assumption of a degener- 
ate mass matrix can be generalised to the non-degenerate case. The universal one-loop effective Lagrangian can then be used without caveats to obtain directly one-loop Wilson coefficients. The advantage of this was demonstrated here in the calculation of the $\bar{c}_{g}$ and $\bar{c}_{\gamma}$ coefficients. One simply takes the mass and $U$ matrices from the quadratic term of the heavy field being integrated out, as defined in (2.1), and substitutes it with the corresponding field strength matrix into the universal expression in (2.4) to get the desired operators, without having to evaluate any loop integrals or match separate calculations in the UV and EFT.

Since the $h g g$ and $h \gamma \gamma$ couplings are loop-induced in the SM, the $\bar{c}_{g}$ and $\bar{c}_{\gamma}$ coefficients are currently the most sensitive to light stops. The stop contribution to these coefficients is also loop-suppressed, thus lowering the EFT cut-off scale, and it is natural to ask at what point the EFT breaks down and the effects of higher-dimensional operators are no longer negligible. We addressed this question by comparing the EFT coefficients with a full calculation in the MSSM, finding that the disagreement is generally $\lesssim 10 \%$ for a lightest stop mass $m_{\tilde{t}_{1}} \gtrsim 500 \mathrm{GeV}$, with the exception of a large $\left|X_{t}\right| \geq 3 m_{\tilde{t}_{1}}$ or accidental cancellations in the Higgs-stop couplings.

The constraints on $\bar{c}_{g}$ and $\bar{c}_{\gamma}$ from a global fit to the current LHC and Tevatron data, and the constraints on $\bar{c}_{T}$ and $\bar{c}_{W}+\bar{c}_{B}$ from LEP electroweak precision observables, were then translated into the corresponding constraints on the stop masses and $X_{t}$. The coefficient $\bar{c}_{g}$ is the most sensitive, followed by $\bar{c}_{T}$, which is equivalent to the oblique $T$ parameter. In the case of degenerate soft masses, this analysis requires $m_{\tilde{t}_{1}} \gtrsim 410 \mathrm{GeV}$ for $X_{t}=0$, and $m_{\tilde{t}_{1}} \gtrsim 200 \mathrm{GeV}$ if we also apply the Higgs mass constraint. This is competitive with direct searches and is complimentary in the sense that it does not depend on how the stop decays. The limits in the non-degenerate case are generally weaker than the Higgs mass requirement, though a few strips in the parameter space compatible with $M_{H}$ can still be excluded.

The sensitivity of future colliders can greatly improve the reach of indirect constraints into the region of parameter space compatible with the observed Higgs mass. The most promising measurements will be the hgg coupling and the $T$ parameter, with FCC-ee capable of reaching a sensitivity to stop masses above $1 \mathrm{TeV}$. Thus, FCC-ee measurements will be able to challenge the naturalness paradigm in a rather model-independent way.

As LHC Run 2 gets under way, the question how to interpret any new physics or lack thereof will be aided by the systematic approach of the ESM. We have demonstrated this for the case of light stops in the MSSM, showing how the EFT framework can simplify both the calculation of relevant observables and the application of experimental constraints on these observables, giving results similar to exact one-loop calculations in the MSSM.

\section{Acknowledgments}

The work of AD was supported by the STFC Grant ST/J002798/1. The work of JE was supported partly by the London Centre for Terauniverse Studies (LCTS), using funding from the European Research Council via the Advanced Investigator Grant 26732, and partly by the STFC Grants ST/J002798/1 and ST/L000326/1. The work of JQ was supported by the STFC Grant ST/L000326/1. The work of TY was supported by a Graduate Teaching Assistantship from King's College London. 
Open Access. This article is distributed under the terms of the Creative Commons Attribution License (CC-BY 4.0), which permits any use, distribution and reproduction in any medium, provided the original author(s) and source are credited.

\section{References}

[1] W. Buchmüller and D. Wyler, Effective Lagrangian Analysis of New Interactions and Flavor Conservation, Nucl. Phys. B 268 (1986) 621 [InSPIRE].

[2] H.D. Politzer, Power Corrections at Short Distances, Nucl. Phys. B 172 (1980) 349 [INSPIRE].

[3] H. Kluberg-Stern and J.B. Zuber, Renormalization of Nonabelian Gauge Theories in a Background Field Gauge. 2. Gauge Invariant Operators, Phys. Rev. D 12 (1975) 3159 [INSPIRE].

[4] C. Grosse-Knetter, Effective Lagrangians with higher derivatives and equations of motion, Phys. Rev. D 49 (1994) 6709 [hep-ph/9306321] [InSPIRE].

[5] C. Arzt, Reduced effective Lagrangians, Phys. Lett. B 342 (1995) 189 [hep-ph/9304230] [INSPIRE].

[6] H. Simma, Equations of motion for effective Lagrangians and penguins in rare B decays, Z. Phys. C 61 (1994) 67 [hep-ph/9307274] [INSPIRE].

[7] J. Wudka, Electroweak effective Lagrangians, Int. J. Mod. Phys. A 9 (1994) 2301 [hep-ph/9406205] [INSPIRE].

[8] T. Appelquist and J. Carazzone, Infrared singularities and massive fields, Phys. Rev. D 11 (1975) 2856.

[9] Z. Han and W. Skiba, Effective theory analysis of precision electroweak data, Phys. Rev. D 71 (2005) 075009 [hep-ph/0412166] [INSPIRE].

[10] T. Corbett, O.J.P. Eboli, J. Gonzalez-Fraile and M.C. Gonzalez-Garcia, Robust Determination of the Higgs Couplings: Power to the Data, Phys. Rev. D 87 (2013) 015022 [arXiv:1211.4580] [INSPIRE].

[11] B. Dumont, S. Fichet and G. von Gersdorff, A Bayesian view of the Higgs sector with higher dimensional operators, JHEP 07 (2013) 065 [arXiv: 1304.3369] [INSPIRE].

[12] M. Ciuchini, E. Franco, S. Mishima and L. Silvestrini, Electroweak Precision Observables, New Physics and the Nature of a 126 GeV Higgs Boson, JHEP 08 (2013) 106 [arXiv: 1306.4644] [INSPIRE].

[13] A. Pomarol and F. Riva, Towards the Ultimate SM Fit to Close in on Higgs Physics, JHEP 01 (2014) 151 [arXiv: 1308.2803] [INSPIRE].

[14] J. de Blas et al., Global Bayesian Analysis of the Higgs-boson Couplings, arXiv:1410.4204 [INSPIRE].

[15] A. Falkowski and F. Riva, Model-independent precision constraints on dimension-6 operators, JHEP 02 (2015) 039 [arXiv: 1411.0669] [INSPIRE].

[16] A. Efrati, A. Falkowski and Y. Soreq, Electroweak constraints on flavorful effective theories, arXiv: 1503.07872 [INSPIRE].

[17] J. Ellis, V. Sanz and T. You, Complete Higgs Sector Constraints on Dimension-6 Operators, JHEP 07 (2014) 036 [arXiv: 1404.3667] [INSPIRE]. 
[18] J. Ellis, V. Sanz and T. You, The Effective Standard Model after LHC Run I, JHEP 03 (2015) 157 [arXiv: 1410.7703] [INSPIRE].

[19] G. Buchalla, O. Catà, A. Celis and C. Krause, Note on Anomalous Higgs-Boson Couplings in Effective Field Theory, arXiv:1504.01707 [INSPIRE].

[20] P. Artoisenet et al., A framework for Higgs characterisation, JHEP 11 (2013) 043 [arXiv: 1306.6464] [INSPIRE].

[21] B. Grzadkowski, M. Iskrzynski, M. Misiak and J. Rosiek, Dimension-Six Terms in the Standard Model Lagrangian, JHEP 10 (2010) 085 [arXiv: 1008.4884] [INSPIRE].

[22] K. Hagiwara, S. Ishihara, R. Szalapski and D. Zeppenfeld, Low-energy effects of new interactions in the electroweak boson sector, Phys. Rev. D 48 (1993) 2182 [INSPIRE].

[23] K. Hagiwara, R. Szalapski and D. Zeppenfeld, Anomalous Higgs boson production and decay, Phys. Lett. B 318 (1993) 155 [hep-ph/9308347] [INSPIRE].

[24] G.J. Gounaris, J. Layssac, J.E. Paschalis and F.M. Renard, Unitarity constraints for new physics induced by dim-6 operators, Z. Phys. C 66 (1995) 619 [hep-ph/9409260] [INSPIRE].

[25] S. Alam, S. Dawson and R. Szalapski, Low-energy constraints on new physics revisited, Phys. Rev. D 57 (1998) 1577 [hep-ph/9706542] [INSPIRE].

[26] R. Barbieri and A. Strumia, What is the limit on the Higgs mass?, Phys. Lett. B 462 (1999) 144 [hep-ph/9905281] [INSPIRE].

[27] V. Barger, T. Han, P. Langacker, B. McElrath and P. Zerwas, Effects of genuine dimension-six Higgs operators, Phys. Rev. D 67 (2003) 115001 [hep-ph/0301097] [INSPIRE].

[28] G.F. Giudice, C. Grojean, A. Pomarol and R. Rattazzi, The Strongly-Interacting Light Higgs, JHEP 06 (2007) 045 [hep-ph/0703164] [INSPIRE].

[29] F. Bonnet, M.B. Gavela, T. Ota and W. Winter, Anomalous Higgs couplings at the LHC and their theoretical interpretation, Phys. Rev. D 85 (2012) 035016 [arXiv:1105.5140] [INSPIRE].

[30] T. Corbett, O.J.P. Eboli, J. Gonzalez-Fraile and M.C. Gonzalez-Garcia, Constraining anomalous Higgs interactions, Phys. Rev. D 86 (2012) 075013 [arXiv:1207.1344] [INSPIRE].

[31] R. Contino, M. Ghezzi, C. Grojean, M. Muhlleitner and M. Spira, Effective Lagrangian for a light Higgs-like scalar, JHEP 07 (2013) 035 [arXiv: 1303.3876] [INSPIRE].

[32] W.-F. Chang, W.-P. Pan and F. Xu, Effective gauge-Higgs operators analysis of new physics associated with the Higgs boson, Phys. Rev. D 88 (2013) 033004 [arXiv:1303.7035] [INSPIRE].

[33] J. Ellis, V. Sanz and T. You, Associated Production Evidence against Higgs Impostors and Anomalous Couplings, Eur. Phys. J. C 73 (2013) 2507 [arXiv:1303.0208] [INSPIRE].

[34] T. Corbett, O.J.P. Éboli, J. Gonzalez-Fraile and M.C. Gonzalez-Garcia, Determining Triple Gauge Boson Couplings from Higgs Data, Phys. Rev. Lett. 111 (2013) 011801 [arXiv: 1304.1151] [INSPIRE].

[35] A. Hayreter and G. Valencia, Constraints on anomalous color dipole operators from Higgs boson production at the LHC, Phys. Rev. D 88 (2013) 034033 [arXiv:1304.6976] [INSPIRE].

[36] H. Mebane, N. Greiner, C. Zhang and S. Willenbrock, Constraints on Electroweak Effective Operators at One Loop, Phys. Rev. D 88 (2013) 015028 [arXiv:1306.3380] [InSPIRE].

[37] M.B. Einhorn and J. Wudka, The Bases of Effective Field Theories, Nucl. Phys. B 876 (2013) 556 [arXiv:1307.0478] [INSPIRE]. 
[38] J. Elias-Miro, J.R. Espinosa, E. Masso and A. Pomarol, Higgs windows to new physics through $D=6$ operators: constraints and one-loop anomalous dimensions, JHEP 11 (2013) 066 [arXiv: 1308.1879] [INSPIRE].

[39] S. Banerjee, S. Mukhopadhyay and B. Mukhopadhyaya, Higher dimensional operators and the LHC Higgs data: The role of modified kinematics, Phys. Rev. D 89 (2014) 053010 [arXiv: 1308.4860] [INSPIRE].

[40] E. Boos, V. Bunichev, M. Dubinin and Y. Kurihara, Higgs boson signal at complete tree level in the SM extension by dimension-six operators, Phys. Rev. D 89 (2014) 035001 [arXiv: 1309.5410] [INSPIRE].

[41] B. Gripaios and D. Sutherland, Searches for CP-violating dimension-6 electroweak gauge boson operators, Phys. Rev. D 89 (2014) 076004 [arXiv:1309.7822] [INSPIRE].

[42] A. Alloul, B. Fuks and V. Sanz, Phenomenology of the Higgs Effective Lagrangian via FEYNRULES, JHEP 04 (2014) 110 [arXiv:1310.5150] [INSPIRE].

[43] C.-Y. Chen, S. Dawson and C. Zhang, Electroweak Effective Operators and Higgs Physics, Phys. Rev. D 89 (2014) 015016 [arXiv:1311.3107] [INSPIRE].

[44] M. Dahiya, S. Dutta and R. Islam, Unitarizing VV Scattering in Light Higgs Scenarios, arXiv: 1311.4523 [INSPIRE].

[45] C. Grojean, E. Salvioni, M. Schlaffer and A. Weiler, Very boosted Higgs in gluon fusion, JHEP 05 (2014) 022 [arXiv: 1312.3317] [INSPIRE].

[46] J. Bramante, A. Delgado and A. Martin, Cornering a hyper Higgs boson: Angular kinematics for boosted Higgs bosons with top pairs, Phys. Rev. D 89 (2014) 093006 [arXiv:1402.5985] [INSPIRE].

[47] R.S. Gupta, A. Pomarol and F. Riva, BSM Primary Effects, Phys. Rev. D 91 (2015) 035001 [arXiv: 1405.0181] [INSPIRE].

[48] J.S. Gainer, J. Lykken, K.T. Matchev, S. Mrenna and M. Park, Beyond Geolocating: Constraining Higher Dimensional Operators in $H \rightarrow 4 \ell$ with Off-Shell Production and More, Phys. Rev. D 91 (2015) 035011 [arXiv: 1403.4951] [inSPIRE].

[49] S. Bar-Shalom, A. Soni and J. Wudka, EFT naturalness: an effective field theory analysis of Higgs naturalness, arXiv:1405.2924 [INSPIRE].

[50] G. Amar et al., Exploration of the tensor structure of the Higgs boson coupling to weak bosons in $e^{+} e^{-}$collisions, JHEP 02 (2015) 128 [arXiv: 1405.3957] [INSPIRE].

[51] A. Azatov, C. Grojean, A. Paul and E. Salvioni, Taming the off-shell Higgs boson, Zh. Eksp. Teor. Fiz. 147 (2015) 410 [arXiv: 1406.6338] [INSPIRE].

[52] E. Masso, An Effective Guide to Beyond the Standard Model Physics, JHEP 10 (2014) 128 [arXiv: 1406.6376] [INSPIRE].

[53] A. Biekötter, A. Knochel, M. Krämer, D. Liu and F. Riva, Vices and virtues of Higgs effective field theories at large energy, Phys. Rev. D 91 (2015) 055029 [arXiv:1406.7320] [INSPIRE].

[54] C. Englert and M. Spannowsky, Effective Theories and Measurements at Colliders, Phys. Lett. B 740 (2015) 8 [arXiv:1408.5147] [INSPIRE].

[55] R. Alonso, E.E. Jenkins and A.V. Manohar, Holomorphy without Supersymmetry in the Standard Model Effective Field Theory, Phys. Lett. B 739 (2014) 95 [arXiv:1409.0868] [INSPIRE]. 
[56] R.M. Godbole, D.J. Miller, K.A. Mohan and C.D. White, Jet substructure and probes of CP-violation in Vh production, JHEP 04 (2015) 103 [arXiv: 1409.5449] [INSPIRE].

[57] M. Trott, On the consistent use of Constructed Observables, JHEP 02 (2015) 046 [arXiv: 1409.7605] [INSPIRE].

[58] F. Goertz, A. Papaefstathiou, L.L. Yang and J. Zurita, Higgs boson pair production in the $D=6$ extension of the SM, JHEP 04 (2015) 167 [arXiv:1410.3471] [INSPIRE].

[59] L. Lehman, Extending the Standard Model Effective Field Theory with the Complete Set of Dimension-7 Operators, Phys. Rev. D 90 (2014) 125023 [arXiv:1410.4193] [INSPIRE].

[60] C. Englert, Y. Soreq and M. Spannowsky, Off-Shell Higgs Coupling Measurements in BSM scenarios, arXiv:1410.5440 [INSPIRE].

[61] A. Devastato, F. Lizzi, C.V. Flores and D. Vassilevich, Unification of coupling constants, dimension 6 operators and the spectral action, Int. J. Mod. Phys. A 30 (2015) 1550033 [arXiv: 1410.6624] [INSPIRE].

[62] D. Ghosh and M. Wiebusch, Dimension-six triple gluon operator in Higgs+jet observables, Phys. Rev. D 91 (2015) 031701 [arXiv:1411.2029] [INSPIRE].

[63] T. Corbett, O. Éboli and M. Gonzalez-Garcia, Unitarity Constraints on Dimension-Six Operators, Phys. Rev. D 91 (2015) 035014 [arXiv:1411.5026] [INSPIRE].

[64] M. Gonzalez-Alonso, A. Greljo, G. Isidori and D. Marzocca, Pseudo-observables in Higgs decays, Eur. Phys. J. C 75 (2015) 128 [arXiv:1412.6038] [InSPIRE].

[65] R. Edezhath, Dimension-6 Operator Constraints from Boosted VBF Higgs, arXiv: 1501.00992 [INSPIRE].

[66] A. Eichhorn et al., The Higgs Mass and the Scale of New Physics, JHEP 04 (2015) 022 [arXiv: 1501.02812] [INSPIRE].

[67] S. Dawson, I.M. Lewis and M. Zeng, Usefulness of effective field theory for boosted Higgs production, Phys. Rev. D 91 (2015) 074012 [arXiv:1501.04103] [INSPIRE].

[68] A. Azatov, R. Contino, G. Panico and M. Son, Effective field theory analysis of double Higgs production via gluon fusion, arXiv:1502.00539 [INSPIRE].

[69] L. Berthier and M. Trott, Towards consistent Electroweak Precision Data constraints in the SMEFT, JHEP 05 (2015) 024 [arXiv: 1502.02570] [INSPIRE].

[70] C. Bobeth and U. Haisch, Anomalous triple gauge couplings from B-meson and kaon observables, arXiv:1503.04829 [INSPIRE].

[71] T. Han, Z. Liu, Z. Qian and J. Sayre, Improving Higgs coupling measurements through ZZ Fusion at the ILC, arXiv:1504.01399 [INSPIRE].

[72] B. Henning, X. Lu and H. Murayama, What do precision Higgs measurements buy us?, arXiv: 1404.1058 [INSPIRE].

[73] B. Henning, X. Lu and H. Murayama, How to use the Standard Model effective field theory, arXiv:1412.1837 [INSPIRE].

[74] N. Craig, M. Farina, M. McCullough and M. Perelstein, Precision Higgsstrahlung as a Probe of New Physics, JHEP 03 (2015) 146 [arXiv:1411.0676] [InSPIRE].

[75] M. Gorbahn, J.M. No and V. Sanz, Benchmarks for Higgs Effective Theory: Extended Higgs Sectors, arXiv:1502.07352 [INSPIRE]. 
[76] S. Willenbrock and C. Zhang, Effective Field Theory Beyond the Standard Model, Ann. Rev. Nucl. Part. Sci. 64 (2014) 83 [arXiv:1401.0470] [INSPIRE].

[77] M.K. Gaillard, The Effective One Loop Lagrangian With Derivative Couplings, Nucl. Phys. B 268 (1986) 669 [INSPIRE].

[78] O. Cheyette, Effective Action for the Standard Model With Large Higgs Mass, Nucl. Phys. B 297 (1988) 183 [INSPIRE].

[79] J. Fan and M. Reece, A New Look at Higgs Constraints on Stops, JHEP 06 (2014) 031 [arXiv: 1401.7671] [INSPIRE].

[80] J. Fan, M. Reece and L.-T. Wang, Possible Futures of Electroweak Precision: ILC, FCC-ee and $C E P C$, arXiv:1411.1054 [INSPIRE].

[81] J. Fan, M. Reece and L.-T. Wang, Precision Natural SUSY at CEPC, FCC-ee and ILC, arXiv: 1412.3107 [INSPIRE].

[82] N. Haba, K. Kaneta, S. Matsumoto and T. Nabeshima, A Simple Method of Calculating Effective Operators, Acta Phys. Polon. B 43 (2012) 405 [arXiv:1106.6106] [InSPIRE].

[83] TLeP Design Study Working Group collaboration, M. Bicer et al., First Look at the Physics Case of TLEP, JHEP 01 (2014) 164 [arXiv:1308.6176] [INSPIRE].

[84] A. Drozd, J. Ellis, J. Quevillon and T. You, niversal One Loop Effective Action for Dimension Six Operators, work in preparation.

[85] A. Djouadi, The Anatomy of electro-weak symmetry breaking. II. The Higgs bosons in the minimal supersymmetric model, Phys. Rept. 459 (2008) 1 [hep-ph/0503173] [InSPIRE].

[86] A. Djouadi et al., The post-Higgs MSSM scenario: Habemus MSSM?, Eur. Phys. J. C 73 (2013) 2650 [arXiv: 1307.5205] [INSPIRE].

[87] A. Djouadi, L. Maiani, A. Polosa, J. Quevillon and V. Riquer, Fully covering the MSSM Higgs sector at the LHC, arXiv: 1502.05653 [INSPIRE].

[88] J.R. Espinosa, C. Grojean, V. Sanz and M. Trott, NSUSY fits, JHEP 12 (2012) 077 [arXiv: 1207.7355] [INSPIRE].

[89] T. Hahn, Generating Feynman diagrams and amplitudes with FeynArts 3, Comput. Phys. Commun. 140 (2001) 418 [hep-ph/0012260] [INSPIRE].

[90] C. Grojean, E.E. Jenkins, A.V. Manohar and M. Trott, Renormalization Group Scaling of Higgs Operators and $\Gamma(h \rightarrow \gamma \gamma)$, JHEP 04 (2013) 016 [arXiv: 1301.2588] [INSPIRE].

[91] J. Elias-Miró, J.R. Espinosa, E. Masso and A. Pomarol, Renormalization of dimension-six operators relevant for the Higgs decays $h \rightarrow \gamma \gamma, \gamma Z$, JHEP 08 (2013) 033 [arXiv:1302.5661] [INSPIRE].

[92] J. Elias-Miro, J.R. Espinosa, E. Masso and A. Pomarol, Higgs windows to new physics through $d=6$ operators: constraints and one-loop anomalous dimensions, JHEP 11 (2013) 066 [arXiv: 1308.1879] [INSPIRE].

[93] E.E. Jenkins, A.V. Manohar and M. Trott, Renormalization Group Evolution of the Standard Model Dimension Six Operators I: Formalism and lambda Dependence, JHEP 10 (2013) 087 [arXiv: 1308.2627] [INSPIRE].

[94] E.E. Jenkins, A.V. Manohar and M. Trott, Renormalization Group Evolution of the Standard Model Dimension Six Operators II: Yukawa Dependence, JHEP 01 (2014) 035 [arXiv:1310.4838] [INSPIRE]. 
[95] R. Alonso, E.E. Jenkins, A.V. Manohar and M. Trott, Renormalization Group Evolution of the Standard Model Dimension Six Operators III: Gauge Coupling Dependence and Phenomenology, JHEP 04 (2014) 159 [arXiv:1312.2014] [INSPIRE].

[96] J. Elias-Miró, C. Grojean, R.S. Gupta and D. Marzocca, Scaling and tuning of $E W$ and Higgs observables, JHEP 05 (2014) 019 [arXiv:1312.2928] [INSPIRE].

[97] R. Alonso, H.-M. Chang, E.E. Jenkins, A.V. Manohar and B. Shotwell, Renormalization group evolution of dimension-six baryon number violating operators, Phys. Lett. B 734 (2014) 302 [arXiv: 1405. 0486] [InSPIRE].

[98] G. Degrassi, S. Heinemeyer, W. Hollik, P. Slavich and G. Weiglein, Towards high precision predictions for the MSSM Higgs sector, Eur. Phys. J. C 28 (2003) 133 [hep-ph/0212020] [INSPIRE].

[99] S. Heinemeyer, W. Hollik and G. Weiglein, The Masses of the neutral CP-even Higgs bosons in the MSSM: Accurate analysis at the two loop level, Eur. Phys. J. C 9 (1999) 343 [hep-ph/9812472] [INSPIRE].

[100] S. Heinemeyer, W. Hollik and G. Weiglein, FeynHiggs: A Program for the calculation of the masses of the neutral CP even Higgs bosons in the MSSM, Comput. Phys. Commun. 124 (2000) 76 [hep-ph/9812320] [INSPIRE].

[101] M. Frank et al., The Higgs Boson Masses and Mixings of the Complex MSSM in the Feynman-Diagrammatic Approach, JHEP 02 (2007) 047 [hep-ph/0611326] [INSPIRE].

[102] http://www.feynhiggs.de.

[103] MasterCode collaboration, K.J. de Vries et al., , KCL-PH-TH/2015-15, LCTS/2015-07, CERN-PH-TH/2015-066 [arXiv: 1504.03260] [INSPIRE].

[104] M.E. Peskin and T. Takeuchi, A New constraint on a strongly interacting Higgs sector, Phys. Rev. Lett. 65 (1990) 964 [INSPIRE].

[105] M.E. Peskin and T. Takeuchi, Estimation of oblique electroweak corrections, Phys. Rev. D 46 (1992) 381 [INSPIRE].

[106] G. Altarelli and R. Barbieri, Vacuum polarization effects of new physics on electroweak processes, Phys. Lett. B 253 (1991) 161 [INSPIRE].

[107] G. Altarelli, R. Barbieri and S. Jadach, Toward a model independent analysis of electroweak data, Nucl. Phys. B 369 (1992) 3 [Erratum ibid. B 376 (1992) 444] [INSPIRE].

[108] TLeP Design Study Working Group collaboration, M. Bicer et al., First Look at the Physics Case of TLEP, JHEP 01 (2014) 164 [arXiv:1308.6176] [INSPIRE].

[109] A. Blondel, Search for heavy right handed neutrinos at circular $e^{+} e^{-}$colliders, at Exploring the Physics Frontier with Circular Colliders, Aspen Colorado U.S.A., 31 January 2015, http://indico.cern.ch/event/336571/other-view?view=standard.

[110] A. Freitas et al., Exploring Quantum Physics at the ILC, arXiv:1307.3962 [INSPIRE].

[111] D.M. Asner et al., ILC Higgs White Paper, arXiv:1310.0763 [InSPIRE].

[112] S. Mishima, Sensitivity to new physics from TLEP precision measurements, at 6th TLEP workshop, CERN, Geneva Switzerland, 16 October 2013, http://indico.cern.ch/event/257713/session/1/contribution/30. 